\section{EMBRYRIDDLE \\ Aeronautical University}

SCHOLARLY COMMONS
International Journal of Aviation, Aeronautics, and Aerospace

$1-1-2017$

\title{
Long and short-range air navigation on spherical Earth
}

Nihad E. Daidzic

AAR Aerospace Consulting, LLC, aaraerospace@cs.com

Follow this and additional works at: https://commons.erau.edu/ijaaa

Part of the Aviation Commons, Geometry and Topology Commons, Navigation, Guidance, Control and Dynamics Commons, Numerical Analysis and Computation Commons, and the Programming Languages and Compilers Commons

\section{Scholarly Commons Citation}

Daidzic, N. E. (2017). Long and short-range air navigation on spherical Earth. International Journal of Aviation, Aeronautics, and Aerospace, 4(1). https://doi.org/10.15394/ijaaa.2017.1160

This Article is brought to you for free and open access by the Journals at Scholarly Commons. It has been accepted for inclusion in International Journal of Aviation, Aeronautics, and Aerospace by an authorized administrator of Scholarly Commons. For more information, please contact commons@erau.edu. 
As the long-range and ultra-long range non-stop commercial flights slowly turn into reality, the accurate characterization and optimization of flight trajectories becomes even more essential. An airplane flying non-stop between two antipodal points on spherical Earth along the Great Circle (GC) route is covering distance of about 10,800 NM $(20,000 \mathrm{~km})$ over-the-ground. Taking into consideration winds (Daidzic, 2014; Daidzic; 2016a) and the flight level (FL), the required air range may exceed 12,500 NM for antipodal ultra-long flights. No civilian or military airplane today (without inflight refueling capability) is capable of such ranges. About 40-60\% increase in air range performance will be required from the future airplanes to achieve truly global range (GR). There is no need to elaborate on the economic aspects of finding the shortest trajectories between two points on Earth. However, many other factors may cause perturbations of such trajectories when considering the minimum-cost, minimum-fuel, or any other goal-function in complex optimizations.

The Earth is not a perfect sphere and due to rotational and gravitational effects the shape is more of an oblate spheroid. Actually, Earth's shape is even more complicated and more appropriately treated in terms of tesseral surface harmonics (Tikhonov and Samarskii, 1990). Earth's Polar radius is about $21 \mathrm{~km}$ shorter than the Equatorial. Several Earth's shape approximations are used:

- Idealized spherical Earth of equivalent volume (implicitly used in International Standard Atmosphere or ISA definition).

- Reference mathematical ellipsoid of revolution (WGS-84, IERS/ITRS). Smooth and oblate.

- Geoid or particular equipotential surface that approximates mean sea-level (MSL). Irregular and locally smooth. Physically the most important measure of Earth's shape. An example of Geoid in use is WGS-84 (revision 2004) EGM96 Geoid.

- Actual or physical Earth surface with all terrain details. This is fractal dimension, scale dependent and mathematically intractable.

Using spherical Earth approximation is sufficient for the majority of longrange air navigation problems. Due to economy of flight, we are particularly interested in the shortest distances between two arbitrary points on Earth. Differential geometry classifies such lines on smooth surfaces as geodesic lines. On the spherical Earth model a geodesic is a Great Circle (GC) or Orthodrome segment. GCs distances are not necessarily always shortest with respect to time as atmospheric wind plays significant role in distance-time optimization problems. The optimization of flight trajectories taking into account atmospheric factors, extended operations (ETOPS) procedures (De Florio, 2016; FAA, 2008), airspace 
restriction, etc., is a difficult task. Finding geodesics on smooth ellipsoidal Earth has been solved. However, finding geodesic between two arbitrary points on the actual Earth surface considering all the vertical terrain features is practically impossible.

Although, it has been with us for many years, the theory of GC and rhumb-line navigation has not been presented clearly and comprehensively for air navigation practitioners, operators, and students. One of the stated purposes of this article is to review and summarize differential geometry and calculus of variation theories as applied to spheres. That will relive readers from searching and consulting multiple sources using different and often confusing terminology.

We are only considering spherical Earth approximation and present theory of GC (Orthodrome geodesic) and rhumb-line (Loxodrome) navigation. For short distances over certain terrestrial regions we also provide some simplified approximate formulas and define their limits of use. Several ultra-long-range navigational problems utilizing existing major international airports are fully solved using Orthodromes and Loxodromes. Graphic representation utilizing Mercator (cylindrical) and azimuthal (planar) projections is presented. The longest commercial non-stop flights today are reaching 8,000 NM (Daidzic, 2014). Increase in air range of, at least, $40 \%$ is required to achieve full GR connecting any two airports on the Earth (Daidzic, 2014). Practically, due to airspace restrictions, ETOPS procedures, and other considerations, the range of existing long-range subsonic airplanes may need to increase by at least $50 \%$.

The main purpose of this article is to give complete and comprehensive consideration of short and long (geodesic) lines on spherical Earth for the purpose of air navigation. While clearly professional navigation planning software is available to major airlines/operators and ATC system it is mostly used as a blackbox. The objective is also to remove mysteries behind the long-range navigational calculations and provide working equations. Spherical approximation is satisfactory for overwhelming number of long-range air navigation problems. In particular, Orthodromes and Loxodromes are typically considered the two most important curves for air navigation. For that purpose we coded working equations into several software platforms (Basic, Fortran, IDL, and Matlab). The main goal of this article was to provide the fundamental theory and understanding, while the computations can be executed in any high-level programming language.

Detailed mathematical derivations are presented in several appendices as to relive a reader interested only in the final results from the heavy mathematical interpretations involving differential geometry, variational calculus, topology and 
other mathematical fields. Indeed, a knowledge of differential geometry, plane and space vectors and vector calculus, and calculus of variation (variational calculus) is required for in-depth understanding of the subject matters. The most important geometric and topological properties of spheres have been reviewed and working equations provided.

An added benefit of presented long-range navigation solutions is relatively easy implementation of the actual Point-of-Equal-Time (PET), Point-of-NoReturn (PNR) and ETOPS limitations for given wind conditions (Daidzic, 2016a) and one-engine-inoperative (OEI) cruising speeds (Daidzic, 2016b). In a future contribution, and for the academic completeness an ellipsoidal Earth model will be introduced with the Great Ellipse (GE) substituting Great Circle (GC). True geodesic computations are complicated even for a smooth ellipsoid of revolution requiring iterative solvers. Generally, GC calculations on spherical Earth are sufficient for reliable air navigation flight planning purposes, considering all other uncertainties involved and mandatory fuel reserves.

Air navigation should be a mandatory course in every professional pilot curriculum and especially so in aviation university education. Unfortunately, it often is not, which results in operational safety degradations. Too much and/or uneducated reliance on sophisticated electronic navigation technology did and certainly will continue to cause aviation accidents and incidents.

\section{Literature review}

A historic account of differential geometry and basic parts used in this work have been consulted from the well-known mathematical classics, such as, Aleksandrov et al. (1999), Goetz (1970), Kreyszig (1964), Lipshutz (1968), Struik (1988), and Wrede (1972). Also more modern books on differential geometry, such as, Oprea (2007) have been consulted. Basic planar and spherical trigonometry theory with calculus applications has been consulted from the books/handbooks by Ayres and Mendelson (2009), Bronstein and Semendjajew (1989), Danby (1962), Dwight (1961), Nielsen and Vanlonkhuyzen (1954), Olza et al. (1974), Spiegel and Liu (1999), and Todhunter (1886). The basic introduction and theory of solid analytic geometry including various lines, planes and curved surfaces was consulted using Hall (1968). A decent short history of mathematical development including the planar and spherical trigonometry is given in Struik (1987). Some special functions, elliptic integrals, and advanced mathematical methods used in navigation, orbital, and celestial calculations is given in classic sources by Abramowitz and Stegun (1984), Byrd and Friedman (1954), Jahnke and Emde (1945), Tikhonov and Samarskii (1990), and Weber and 
Arfken (2004). The general theory of calculus of variations and its applications in analytical mechanics and geodesics on the sphere are given in, for example, Dym and Shames (2013), Fox (1987), Greenwood (1987), Lanzos (1986), Lass (2009), Smith (1998), Widder (1989), etc. An introduction into geodesy and geodetic computations was consulted from the well-known classics such as Bomford (1983), Torge (2001), and Vaníček and Krakiwsky (1986).

Basic principles of marine and air navigation and navigational instrumentation are given in books by Bowditch's bicentennial edition (2002), Bradley (1942), De Remer and McLean (1998), Jeppesen (2007), Tooley and Wyatt (2007), Underdown and Palmer (2001), and Wolper (2001). However, none of these sources except maybe to an extent Wolper go into any deeper air navigation mathematical theory and calculation procedures. Sinnott (1984) proposed the use of the, so called, haversine formula for the GC navigation problems on spherical Earth due to problems with numerical accuracy using the classic cosine-formula for small central angles. Williams (2011) provides many useful aviation formulas, but the equations are written as pseudo-language mathematical expressions, difficult to read, and no background information is provided. Phillips (2004) provides algorithms for GC and rhumb-line navigation, but without derivations. Tewari (2007) presents GC and long-range airplane flight computations and demonstrates how in the absence of wind with no yawing and rolling motion, the airplane will actually follow a GC route. Recently, Weintrit and Kopacz (2011) presented a novel approach to Loxodrome, Orthodrome, and general geodesic problems in Electronic Chart Display and Information System (ECDIS) used for nautical navigation.

Basic GC navigation theory also finds many applications is celestial navigation, orbital mechanics, and astronomy and we used sources such as, Bate et al. (1971) and Fitzpatrick (2012) for some useful information. Geodetic theory and computations with geometric geodesy and geodetic datums on reference terrestrial ellipsoid with some historical accounts has been provided in reports by Jekeli (2012), Krakiwsky and Thomson (1974), Rapp (1991), and Rapp (1993). Many geodetic computations also find applications in geophysics and we mention some better known sources such as Lowrie (2007) that deal with various aspects of geodetic geometry, definitions, and computations.

A good review of rhumb-line calculations on a sphere is given by Alexander (2004) and for terrestrial ellipsoid by Williams (1950). Kos et al (1999) derived and solved differential equation of Loxodrome on spherical Earth using difference of co-latitudes to find its length. GE theory with geodesic and rhumb-line calculations on spheroidal (ellipsoidal) Earth were given by Bennett 
(1996), Bowring (1984), Sjöberg (2012), Tseng and Lee (2010), and Williams (1996). A true numerical geodesic computations for direct and inverse geodesic problems on ellipsoid of revolution (terrestrial spheroid) is given in a contribution by Vincenty (1975). Consideration of elliptic integrals used in geodesic problems was recently addressed by Rollins (2010). More recently Karney (2013) presented a comprehensive account of algorithms for geodesics.

Motion of aircraft in an inertial frame of reference and non-inertial topocentric frames was consider by Miele (2016). McIntyre (2000) provides in depth considerations of motion on a rotating sphere. Fitzpatrick (2012) gives good account of inertial and non-inertial frames of references on Earth. Discussion of airplane trajectories and consideration of apparent forces in various non-inertial frames of references during GC flights will be addressed in a future contribution.

\section{Great Circle and Rhumb-line Navigation on Spherical Earth}

Most often the inverse geodetic problem will be solved where the geodetic (geographic) spherical coordinates of departure and arrival (destination) airports are given. Sufficiently complete theory on fundamental geometric and topological properties of spherical Earth is given in Appendix A. Derivation of geodesic lines on spherical Earth, i.e., Orthodromes (GC arcs) is presented in Appendix B. The GC arcs lie in an osculating plane that contains Earth's center. A proof that GC arcs are indeed shortest distances on spherical Earth is presented. Long distance GC navigation on spherical Earth is discussed in Appendix C. The working equations for calculating no-wind true courses (TC) and the vertex properties are derived. GC and rhumb-line routes are plotted in cylindrical conformal Mercator and/or polar Orthographic projections. Short-distance GC formulas were derived in Appendix D. Additionally, stereographic, gnomonic and orthographic polar (azimuthal) projections have been introduced. Theory of rhumb-line navigation on spherical Earth is presented in Appendix E.

The traditional geographic latitude/longitude coordinates are first converted into truncated angular degree form by using (N+, S-, E+, W-):

DDD MMSS.SSSS $=D D D+\frac{M M}{60}+\frac{S S \cdot S S S S}{3,600}=D D D \cdot D D D D D D D$

Geographic coordinates with an accuracy of one angular second $\left(2.78 \times 10^{-4}\right.$ degree), provides an accuracy of about $100 \mathrm{ft}(30 \mathrm{~m})$. A hundredth of an angular second delivers navigational uncertainty of about $1 \mathrm{ft}(0.3 \mathrm{~m})$. Eight significant digits representation is thus sufficiently accurate for air-navigation applications. 
Typically, aircraft fly at constant pressure altitudes, which at high altitudes is referenced to a standard pressure datum (29.92 inch $\mathrm{Hg}$ or $1013.25 \mathrm{hPa}$ ). If Earth's oblateness is neglected and spatial changes of atmospheric pressure it can be said that airplanes fly in concentric circles around Earth's center. In that case any GC will have arc-length of about 21,600 NM (or about 40,000 km or 25,000 $\mathrm{SM})$. Future ultra-long range airplane should be able to fly non-stop half of any GC to a point which is exactly opposite on the Earth surface (antipodal or conjugate point) to achieve global range (Daidzic, 2014). Between two antipodal points there are infinitely many GCs all of which have equal length assuming spherical earth. The actual terrain elevation is irrelevant in high-altitude cruise flight. Orthometric or Mean Seal Level (MSL) altitude is given in reference to local Geoid height. Terrain elevation can be given in respect to vertical datum contained in WGS 84 spheroid (GPS reference ellipsoid) and when corrected for local Geoid height yields orthometric height. More accurate trajectory calculations should also account for orthometric altitude changes due to variable air pressure, but such considerations may not be significant for majority of flights. Distance errors due to the actual shape of the Earth are less than $0.5 \%$ and often within $0.3 \%$ and thus practically insignificant for most cases.

Utilizing the spherical Law of Cosines (Appendix C) and the average Earth radius $\bar{R}_{E}(=6,371,000 \mathrm{~m})$ plus the average cruising altitude $\bar{h}$, the actual GC arc distance between two surface points $P_{1}\left(\phi_{1}, \lambda_{1}\right)$ and $P_{2}\left(\phi_{2}, \lambda_{2}\right)$ becomes:

$$
O_{1-2}=\bar{R}_{E} \cdot\left[1+\left(\bar{h} / \bar{R}_{E}\right)\right] \cdot \cos ^{-1}\left[\cos \phi_{1} \cdot \cos \phi_{2} \cdot \cos (\Delta \lambda)+\sin \phi_{1} \cdot \sin \phi_{2}\right]
$$

An alternative $\mathrm{GC}$-arc distance formula using the trigonometric haversine function (Appendix C) yields:

$$
\begin{aligned}
& O_{1-2}=2 \cdot \bar{R}_{E} \cdot\left[1+\left(\bar{h} / \bar{R}_{E}\right)\right] \cdot \sin ^{-1}\left[\sqrt{\sin ^{2}\left(\frac{\Delta \phi}{2}\right)+\cos \phi_{1} \cdot \cos \phi_{2} \cdot \sin ^{2}\left(\frac{\Delta \lambda}{2}\right)}\right]= \\
& =2 \cdot \bar{R}_{E} \cdot\left[1+\left(\bar{h} / \bar{R}_{E}\right)\right] \cdot \tan ^{-1}\left\{\frac{\sqrt{\text { havers }(\Delta \phi)+\cos \phi_{1} \cdot \cos \phi_{2} \cdot \text { havers }(\Delta \lambda)}}{\left.\sqrt{1-\left[\text { havers }(\Delta \phi)+\cos \phi_{1} \cdot \cos \phi_{2} \cdot \text { havers }(\Delta \lambda)\right.}\right]}\right\}
\end{aligned}
$$

Adding the average cruising altitude, or the maximum planned cruising altitude, does not affect GC distance very much (10-20 NM), but is a conservative estimate reducing uncertainties. All courses, vertex properties, and GC and rhumb-line waypoints were calculated using expressions derived in appendices. The geographical coordinates are given as latitudes $\phi(\mathrm{N}+, \mathrm{S}-)$ and longitudes $\lambda$ (W-, E+) for desired airport pairs on spherical Earth. The geodetic coordinates of 
some major international airports used in program testing and route calculations are given in Table 1 .

Table 1

Airports used in long- and ultra-long range route computations and testing

\begin{tabular}{|c|c|c|c|c|}
\hline ICAO & IATA & City & $\begin{array}{l}\text { Latitude (N+/S-) } \\
\text { DD MM SS.SSSS }\end{array}$ & $\begin{array}{l}\text { Longitude (E+/W-) } \\
\text { DDD MM SS.SSSS }\end{array}$ \\
\hline \multirow{2}{*}{ ZBAA } & \multirow{2}{*}{ PEK } & \multirow{2}{*}{$\begin{array}{l}\text { Beijing, } \\
\text { China }\end{array}$} & $\mathrm{N} 400400.0000$ & E 1163600.0000 \\
\hline & & & +40.08000000 & +116.58444444 \\
\hline \multirow{2}{*}{ ZSPD } & \multirow{2}{*}{ PVD } & \multirow{2}{*}{$\begin{array}{l}\text { Shanghai, } \\
\text { China }\end{array}$} & N 310900.0000 & E 1214800.0000 \\
\hline & & & +31.1500000 & +121.8000000 \\
\hline \multirow{2}{*}{ SAEZ } & \multirow{2}{*}{ EZE } & \multirow{2}{*}{$\begin{array}{c}\text { Buenos Aires, } \\
\text { Argentina }\end{array}$} & S 344920.0000 & W 0583209.0000 \\
\hline & & & -34.822222222 & -58.53583333 \\
\hline \multirow{2}{*}{ RJAA } & \multirow{2}{*}{ NRT } & \multirow{2}{*}{$\begin{array}{l}\text { Tokyo-Narita, } \\
\text { Japan }\end{array}$} & N 354555.0000 & E 1402308.0000 \\
\hline & & & +35.765278 & +140.385556 \\
\hline \multirow{2}{*}{ SBGL } & \multirow{2}{*}{ GIG } & \multirow{2}{*}{$\begin{array}{c}\text { Rio de Janerio, } \\
\text { Brazil }\end{array}$} & S 224832.0000 & W 0431437.0000 \\
\hline & & & -22.808902 & -43.243646 \\
\hline \multirow{2}{*}{ KSEA } & \multirow{2}{*}{ SEA } & \multirow{2}{*}{$\begin{array}{l}\text { Seattle, WA, } \\
\text { United States }\end{array}$} & $\mathrm{N} 472700.0000$ & W 1221842.0000 \\
\hline & & & +47.449889 & -122.311777 \\
\hline \multirow{2}{*}{ EGGL } & \multirow{2}{*}{ LHR } & \multirow{2}{*}{$\begin{array}{c}\text { London, England, } \\
\text { UK }\end{array}$} & N 512839.0000 & W 0002741.0000 \\
\hline & & & +51.477500 & -0.461388 \\
\hline \multirow{2}{*}{ YSSY } & \multirow{2}{*}{ SYD } & \multirow{2}{*}{ Sydney, Australia } & S 335646.0000 & E 1511038.0000 \\
\hline & & & -33.946110 & +151.177222 \\
\hline \multirow{2}{*}{ FAOR } & \multirow{2}{*}{ JNB } & \multirow{2}{*}{$\begin{array}{l}\text { Johannesburg, } \\
\text { South Africa }\end{array}$} & S 260801.0000 & E 0281432.0000 \\
\hline & & & -26.133693 & 28.242317 \\
\hline \multirow{2}{*}{ MMMX } & \multirow{2}{*}{ MEX } & \multirow{2}{*}{$\begin{array}{l}\text { México City, } \\
\text { México }\end{array}$} & N 192611.0000 & W 0990420.0000 \\
\hline & & & +19.436303 & -99.072096 \\
\hline & & Kuala Lumpur, & $\mathrm{N} 024444.0000$ & E 1014236.0000 \\
\hline WMKK & $\mathrm{KUL}$ & Malaysia & +2.745578 & +101.709917 \\
\hline UUFF & IO & Moscow, Russian & N 555821.0000 & E 0372447.0000 \\
\hline UUEE & Sve & Federation & +55.972500 & +37.413056 \\
\hline & & Colombo, Sri & N 071051.0000 & E 0795303.0000 \\
\hline VCBI & $\mathrm{CMIB}$ & Lanka & +7.180756 & +79.884117 \\
\hline SFOM & UIO & Quito, Pichincha, & S 000648.0000 & W 0782131.0000 \\
\hline SEQIM & 010 & Ecuador & -0.113332 & -78.358610 \\
\hline & & Sarajevo, Bosnia & $\mathrm{N} 434929.0000$ & E 0181953.0000 \\
\hline LQSA & $\mathrm{SJJ}$ & & +43.82472222 & +18.33138889 \\
\hline & & Minneapolis-STP, & N 445255.0000 & W 0931318.0000 \\
\hline KIVSP & MSP & MN, United States & +44.88194444 & -93.22166667 \\
\hline
\end{tabular}


Rhumb-line or Loxodrome-arc distance of constant course angle $\alpha$ on spherical Earth is calculated as (Appendix E):

$L_{1-2}=\bar{R}_{E} \cdot\left[1+\left(\bar{h} / \bar{R}_{E}\right)\right] \cdot\left|\frac{\phi_{2}-\phi_{1}}{\cos \alpha}\right|=\bar{R}_{E} \cdot\left[1+\left(\bar{h} / \bar{R}_{E}\right)\right] \cdot\left|\phi_{2}-\phi_{1}\right| \cdot|\sec \alpha|$

Special care has to be taken to convert spherical angles into Earth-based headings 0-360 degrees. Attention also was warranted when crossing the prime and its anti-meridian or practically the International Date Line (IDL). The cyclic non-unique nature of trigonometric functions creates many problems when performing calculations as illustrated in Figures 1 and 2. The main navigation program originally developed in the True Basic v.5.5 contains subroutines that inspect each airport location and then calculate route waypoints and courses.

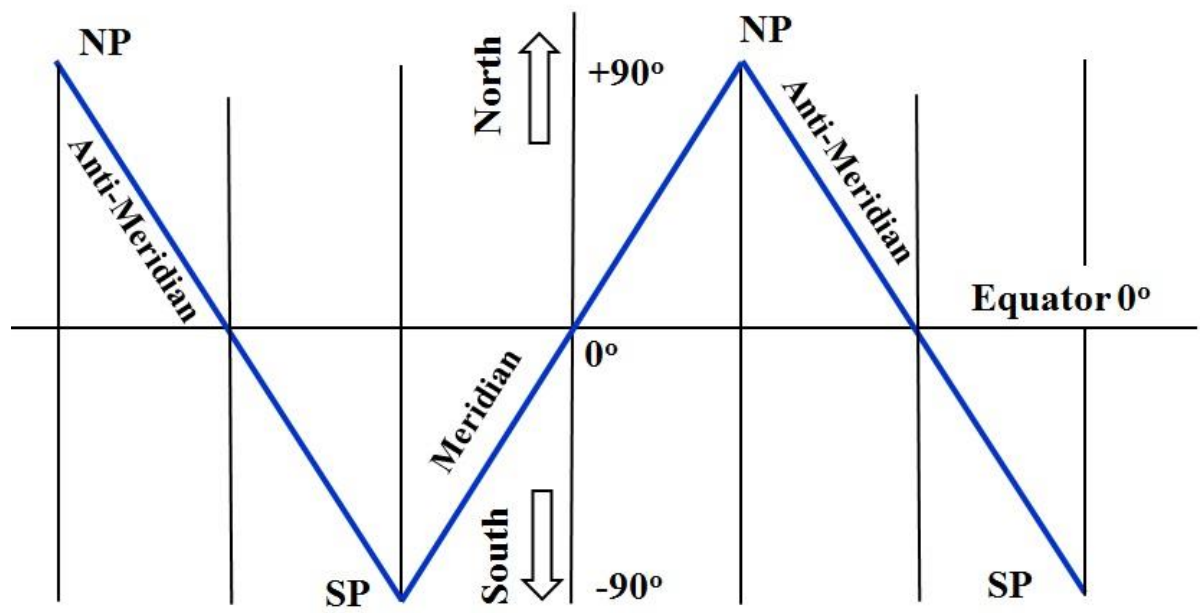

Figure 1. Change of latitude on spherical Earth model. Not to scale.

Navigational calculations were performed on a multi-core 64-bit floatingpoint CPU to minimize rounding errors. True Basic v.5.5 and v.6 (64-bit), 32-bit optimizing-compiler Lahey Fortran 90/95 (Incline Village, NV), 64-bit optimizing Absoft Fortran 90/95 (Troy, MI) with many 2003/2008 extensions, and Matlab R2015a (ver. 8.5, Mathworks, Natick, MA) codes were developed with the graphical capabilities showing GC and Rhumb-line routes on Cylindrical Mercator and Polar Orthographic projections. Summary of some tested long-range route computations using loxodromic and orthodromic navigation is given in Table 2. Most GC (no rhumb-line) graphical results were generated using the Great Circle Mapper ${ }^{\odot}$, copyright Karl L. Swartz (www.gcmap.com). Our 
graphical capabilities are currently modest, but powerful visual representations using IDL (Interactive Data Language) mapping capabilities will be available soon. Several ultra-long routes between some major International airports were used to test and demonstrate the capability and the accuracy of our NAV solvers. Moreover, the intention was to highlight the difficulties and the curiosities when navigating on Earth. Our AARNAV ${ }^{\mathrm{TM}}$ navigation programs used spherical-Earth approximation, while the GC Mapper ${ }^{\circledR}$ calculator uses geodesic calculations on oblate Earth. We also used the marine navigation www.Onboardintelligence.com calculator to independently test and verify GC and rhumb-line results.

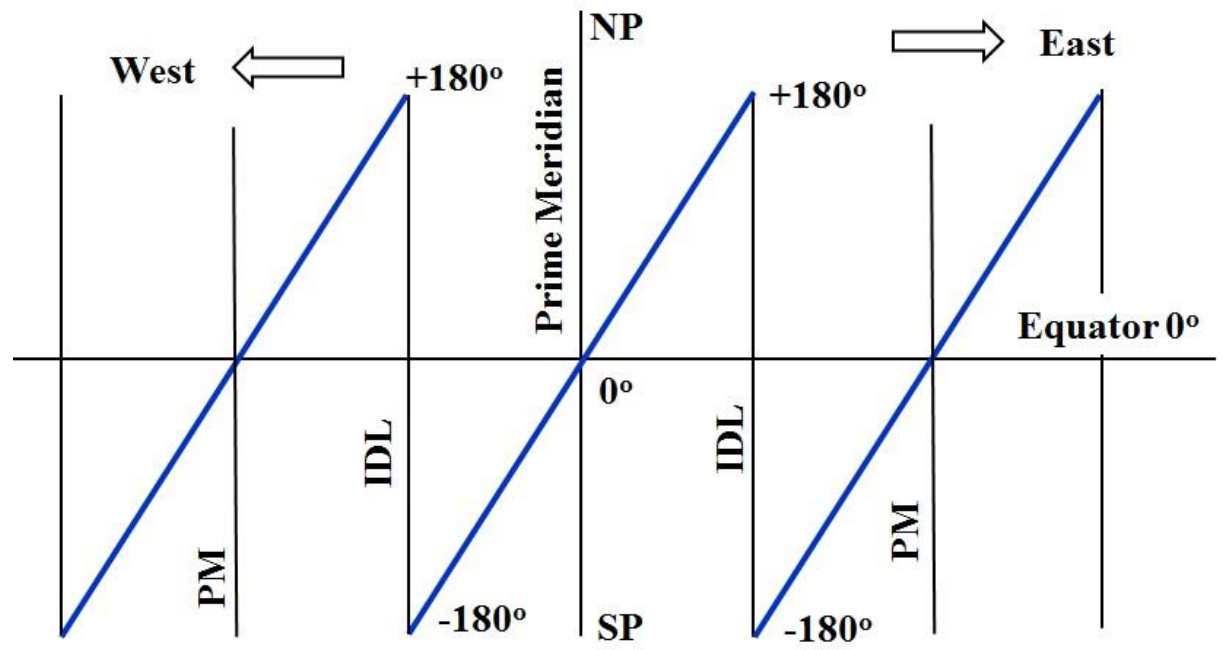

Figure 2. Change of longitude on spherical Earth model. Not to scale.

\section{Results and discussion}

Let us first consider a short flight from ZBAA to ZSPD. Interestingly, Beijing (ZBAA) and Shanghai (ZSPD) are only 594.38 NM orthodromic-distance apart (at FL360) with the Shanghai being S-SE of Beijing (numerical results are summarized in Table 2). The ZBAA orthodrome departure course of $153.084^{\circ}$ and the ZSPD arrival course is $156.127^{\circ}$ (see Table 2). The loxodrome constant course and distance are $154.689^{\circ}$ and 594.45 NM respectively (only about $0.07 \mathrm{NM}$ or about $426 \mathrm{ft}$ longer). The vertex (Lat/Long) of the ZBAA to ZSPD GC-route is located outside of the arc segment at N69.73/E044.69.

The first long-range route we discuss is the route between the SAEZ and ZBAA (Daidzic, 2014). The Orthodrome distance flying at 36,000 ft delivers distance of $10,433.26 \mathrm{NM}$. The initial outbound heading (from SAEZ) is $34.92^{\circ}$ 
and the final inbound heading (at ZBAA) is $142.11^{\circ}$ with the northerly vertex of $\mathrm{N} 61.97^{\circ}$ at E053.20 $0^{\circ}$ longitude. The total change in heading is about $107^{\circ}$. The illustration of the GC route is shown in Figure 3. GC Mapper ${ }^{\odot}$ oblate-Earth (WGS-84) calculator returned the geodesic distance within $2.3 \mathrm{NM}$ of our orthodrome computations. The geodesic route overflies eastern parts of Brazil, crosses Atlantic ocean northbound and tracks parallel to the coast of western Africa, skimming N-W Europe and N-W portions of Russia and then after reaching vertex it "descends" over Mongolia to Beijing on SE headings.

Table 2

Long-distance Loxodromic $\left(L_{1-2}\right)$ and Orthodromic $\left(O_{1-2}\right)$ routes at FL 360

\begin{tabular}{ccccccc} 
Route & $\mathbf{L}_{\mathbf{1 - 2}}[\mathbf{N M}]$ & $\alpha_{L}$ & $\mathbf{O}_{\mathbf{1 - 2}}[\mathbf{N M}]$ & $\alpha_{1}$ & $\alpha_{2}$ & $\begin{array}{c}\text { Vertex } \\
\text { Lat. }\end{array}$ \\
\hline ZBAA-ZSPD & 594.45 & 154.69 & 594.38 & 153.08 & 156.13 & N69.73 \\
\hline SAEZ-ZBAA & $10,730.47$ & 65.18 & $10,433.26$ & 34.92 & 142.11 & N61.97 \\
\hline SAEZ-ZSPD & $10,930.39$ & 291.28 & $10,604.11$ & 184.38 & 355.80 & S86.41 \\
\hline SBGL-RJAA & $10,656.37$ & 289.31 & $10,023.92$ & 347.13 & 194.66 & N78.15 \\
\hline SEQM-WMKK & $10,819.16$ & 270.91 & $10,667.53$ & 358.51 & 181.49 & N88.51 \\
\hline KSEA-FAOR & $9,329.08$ & 118.32 & $8,934.82$ & 57.79 & 140.41 & N55.10 \\
\hline EGGL-YSSY & $9,578.70$ & 122.44 & $9,206.03$ & 60.46 & 139.22 & N57.19 \\
\hline MMMX-WMKK & $9,414.94$ & 263.88 & $9,012.50$ & 315.12 & 221.77 & N48.29 \\
\hline MMMX-VCBI & $10,477.80$ & 94.03 & $9,223.85$ & 2.31 & 177.80 & N87.82 \\
\hline LQSA-KMSP & $4,797.61$ & 270.76 & $4,359.97$ & 316.29 & 224.72 & N60.10 \\
\hline
\end{tabular}

The rhumb-line distance SAEZ-ZBAA is 10,730.47 NM (Loxodrome is about $297.22 \mathrm{NM}$ or $2.85 \%$ longer than Orthodrome) on a constant heading of about $65.18^{\circ}$ and includes about 3,500 NM flight over the dangerous southern Atlantic Ocean. Rudimentary graphs were superimposed on a publically-available conformal Mercator projection image of the world as shown in Figure 4 (Latitudes are unevenly spaced as $\pm 15, \pm 30, \pm 45, \pm 60$, and \pm 75 degrees). The GC (red solid line) and rhumb-line (blue solid line) routes are clearly discernable. The apparently shorter rhumb-line is quite deceptive due to stretching of the scale at higher latitudes on conformal Mercator projection. The difference between the GC route and the true Geodesic using GC Mapper is very small. 


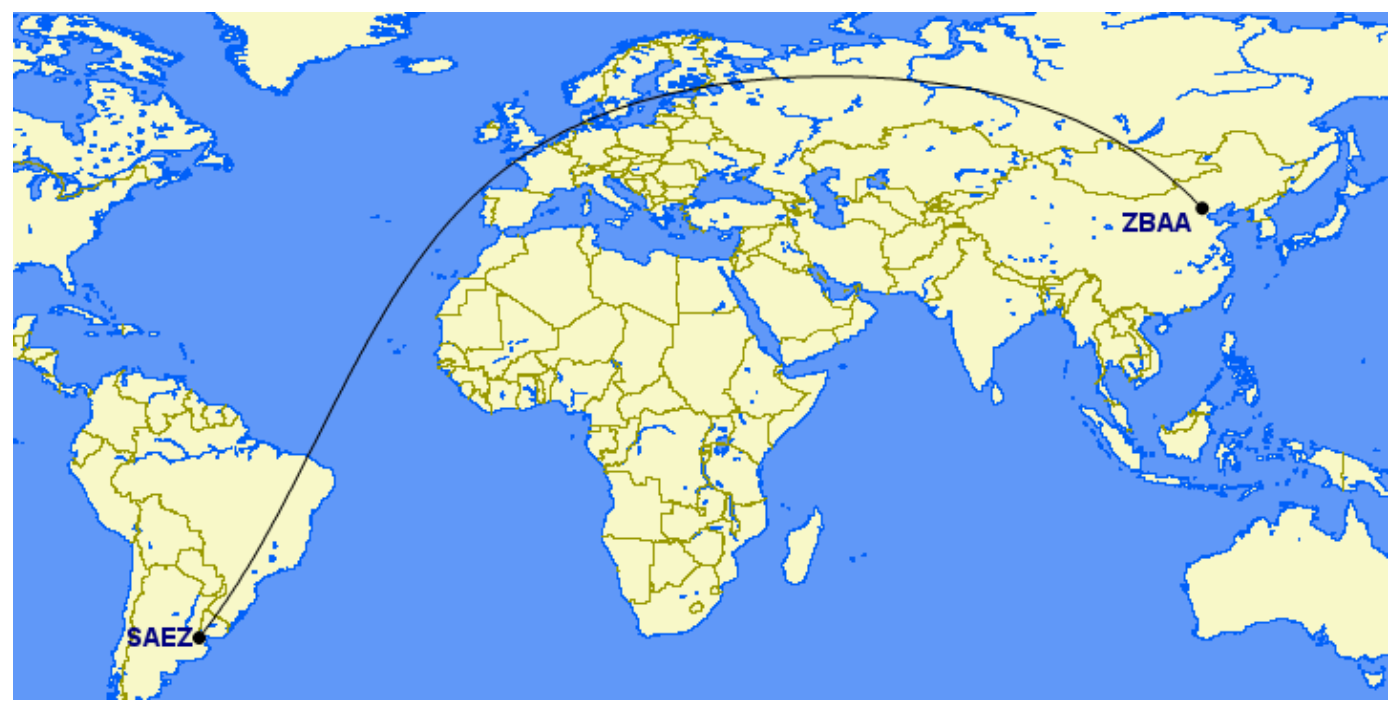

Figure 3. Great circle (geodesic) route SAEZ to ZBAA (EZE to PEK) on conformal cylindrical Mercator chart. Courtesy of GC Mapper. Maps generated by the Great Circle Mapper (www.gcmap.com) - copyright @ Karl L. Swartz.

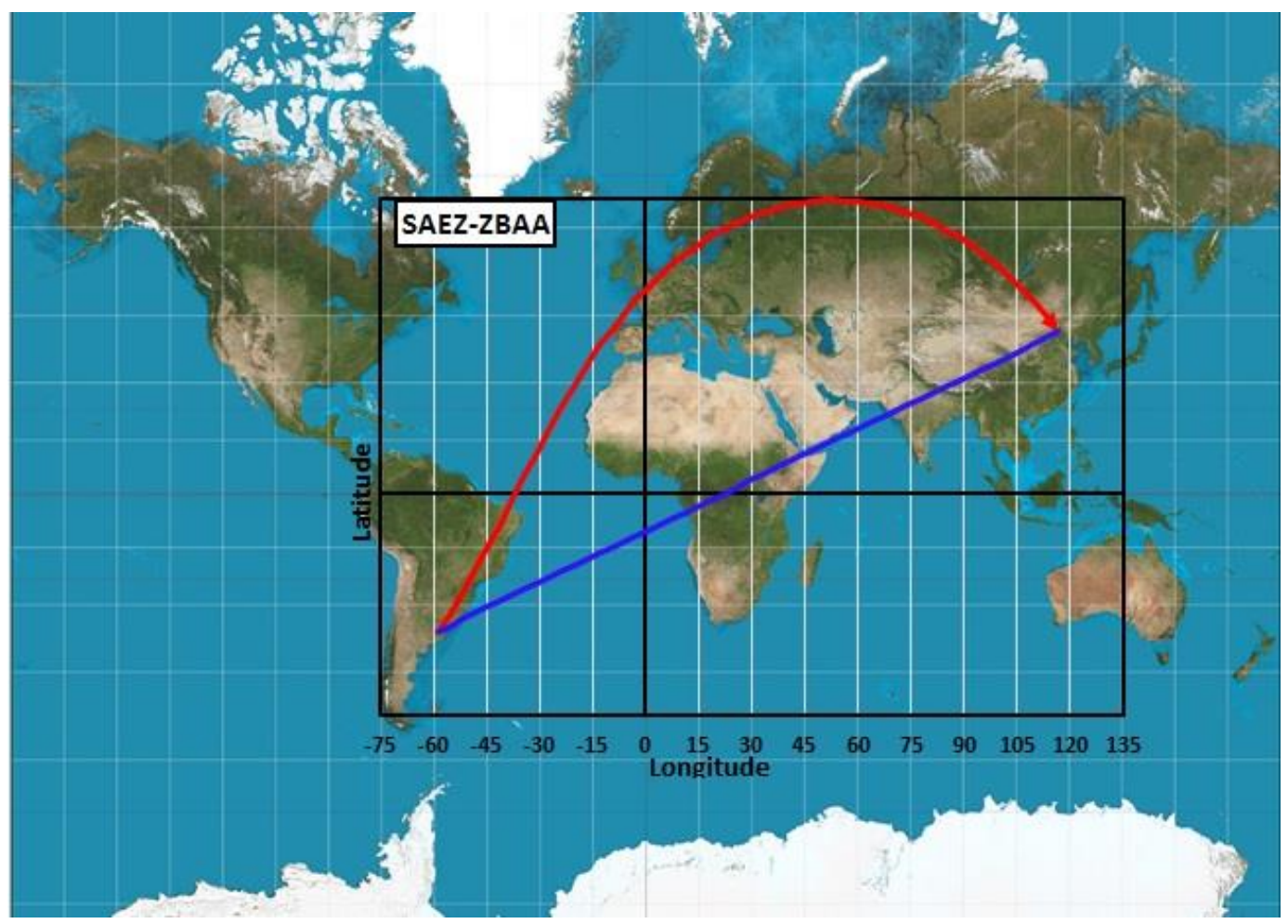

Figure 4. GC route SAEZ to ZBAA on conformal cylindrical Mercator chart. 
The long-range route from SAEZ to ZSPD is illustrated in Figure 5 using GC Mapper ${ }^{\odot}$ and Mercator cylindrical projections. The same flight is partially also shown in Figure 6 using Polar Orthographic projection. In the case of SAEZZSPD, the shortest (orthodrome) distance at FL360 is over Antarctica (SP) at 10,604.11 NM and a mere 200 NM short of antipodal distance.

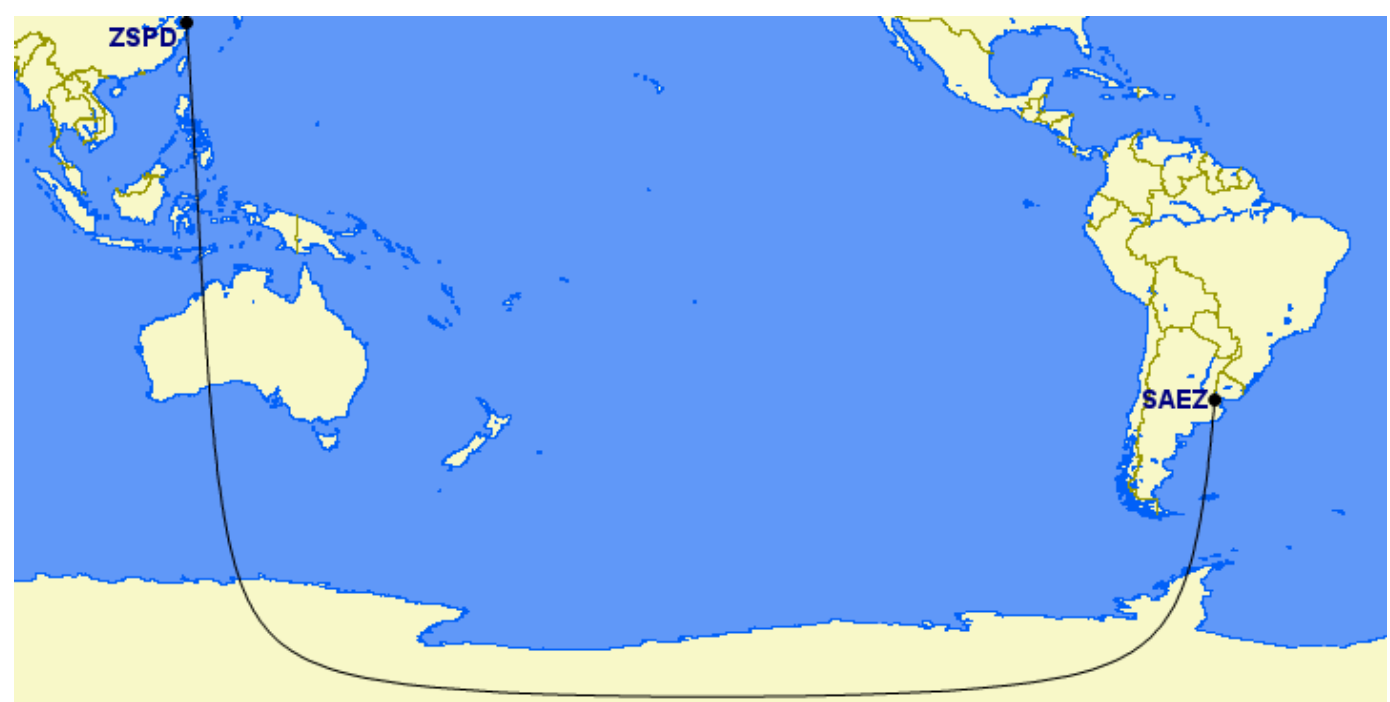

Figure 5. Geodesic route SAEZ to ZSPD on conformal cylindrical Mercator chart. Courtesy of GC Mapper. Maps generated by the Great Circle Mapper (www.gcmap.com) - copyright @ $($ Karl L. Swartz.

The initial outbound heading from SAEZ is now almost straight south or about $184^{\circ}$, while the final inbound heading to destination ZSPD is about $356^{\circ}$. The vertex is very close to SP at $-86.41^{\circ}$. The rhumb-line distance is $10,930.39$ $\mathrm{NM}$ at the constant heading of $291.28^{\circ}$ (see Table 2) and involves diagonal flight over the entire Pacific Ocean. The GC Mapper returned the value of 10,580 NM (over surface) with the departure heading of $183.9^{\circ}$. None of the two SAEZ-ZSPD routes (GC or rhumb-line) are particularly friendly in terms of ETOPS procedures as they involve long flights over Polar Regions and oceans. What is very interesting is that while destinations ZBAA and ZSPD are very close to each other, the routes from SAEZ could not be more different. One reaches high northern latitudes, while the other goes almost straight over the South Pole (SP) and straight across Antarctica.

If such a direct non-stop flight is ever planned it would be perhaps better in terms of safety and following the ETOPS procedures to follow similar route as in SAEZ-ZBAA route, i.e., fly over the South- and the North-America, passing 
close to the North Pole (NP) and approach ZSPD on an almost straight south course. Such longer route would probably add another $1 \frac{1 / 2}{2}$ hour of flight, but there would be more options for deviations and alternates. Thus to have truly global range an airplane will have to have practical ground range exceeding half of the Earth circumference (e.g., 12,000 NM over ground) in which case the required air range would likely exceed 13,000 NM.

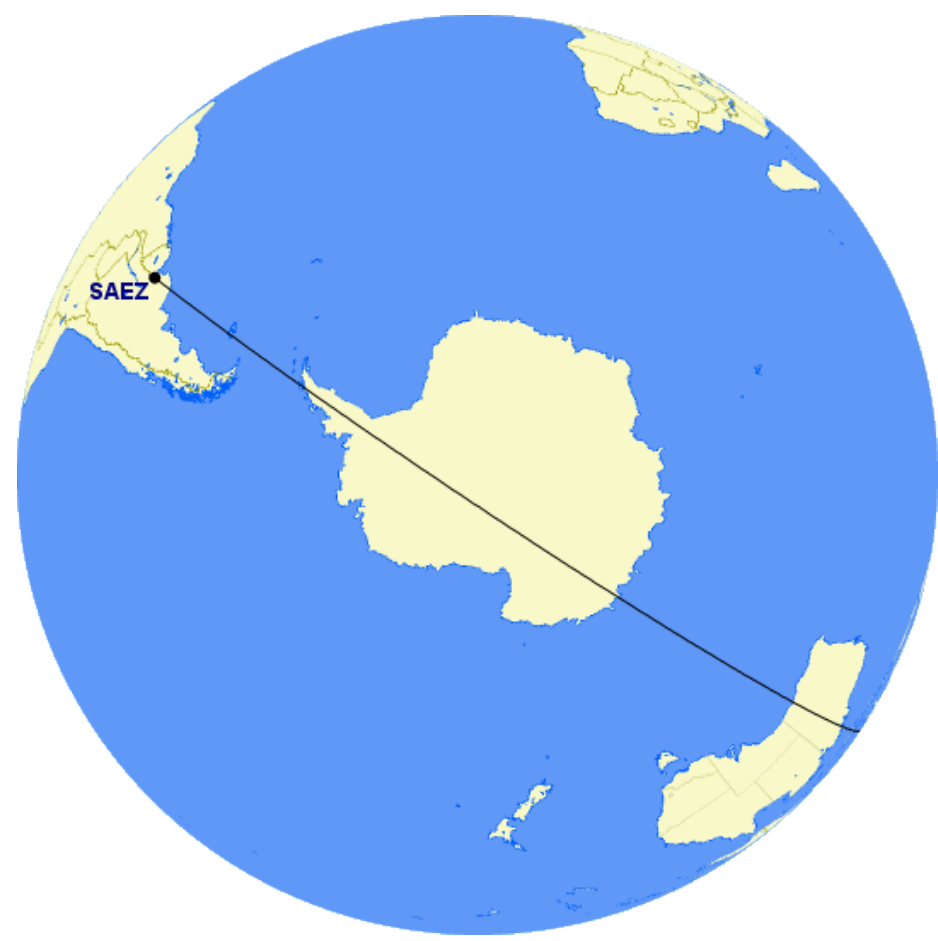

Figure 6. SAEZ to ZSPD geodesic route on Polar Orthographic chart. Courtesy of GC Mapper. Maps generated by the Great Circle Mapper (www.gcmap.com) copyright $\odot$ Karl L. Swartz.

The next possible future ultra-long range flight specifically considered is from Rio de Janerio (Brazil) SBGL (GIG) to Tokyo Narita in Japan RJAA (NRT). The route is shown in Figure 7 on Mercator chart and in Figure 8 on Polar orthographic chart using GC Mapper ${ }^{\odot}$. According to Table 2's summary of several ultra-long routes, we have the rhumb-line distance of 10,656 NM on a straight W-NW course of $289.31^{\circ}$ while the GC distance with average cruise altitude of 36,000 ft delivers 10,023.92 NM. The differences between the GC and rhumb-line is quite significant at about $632.45 \mathrm{NM}$. Our calculator returned $347.13^{\circ}$ for outbound and $194.66^{\circ}$ for inbound course. Our calculations estimated vertex at $+78.15^{\circ}$ latitude (North). We also utilized Onboardintelligence.com 
(Onboard Marine navigation software) calculator and obtained 10,001.52 NM for Orthodrome (over surface) with the departure heading of $347.4^{\circ}$ and the inbound course into Narita of $194.4^{\circ}$. Its vertex calculations resulted in $+78^{\circ} 24^{\prime} .15$ latitude and $-138^{\circ} 09^{\prime} .22$ longitude. Similar results were also obtained using the GC Mapper ${ }^{\odot}$, which returned $10,004 \mathrm{NM}$ and $347.5^{\circ}$ geodesic over the reference ellipsoidal surface (WGS-84).

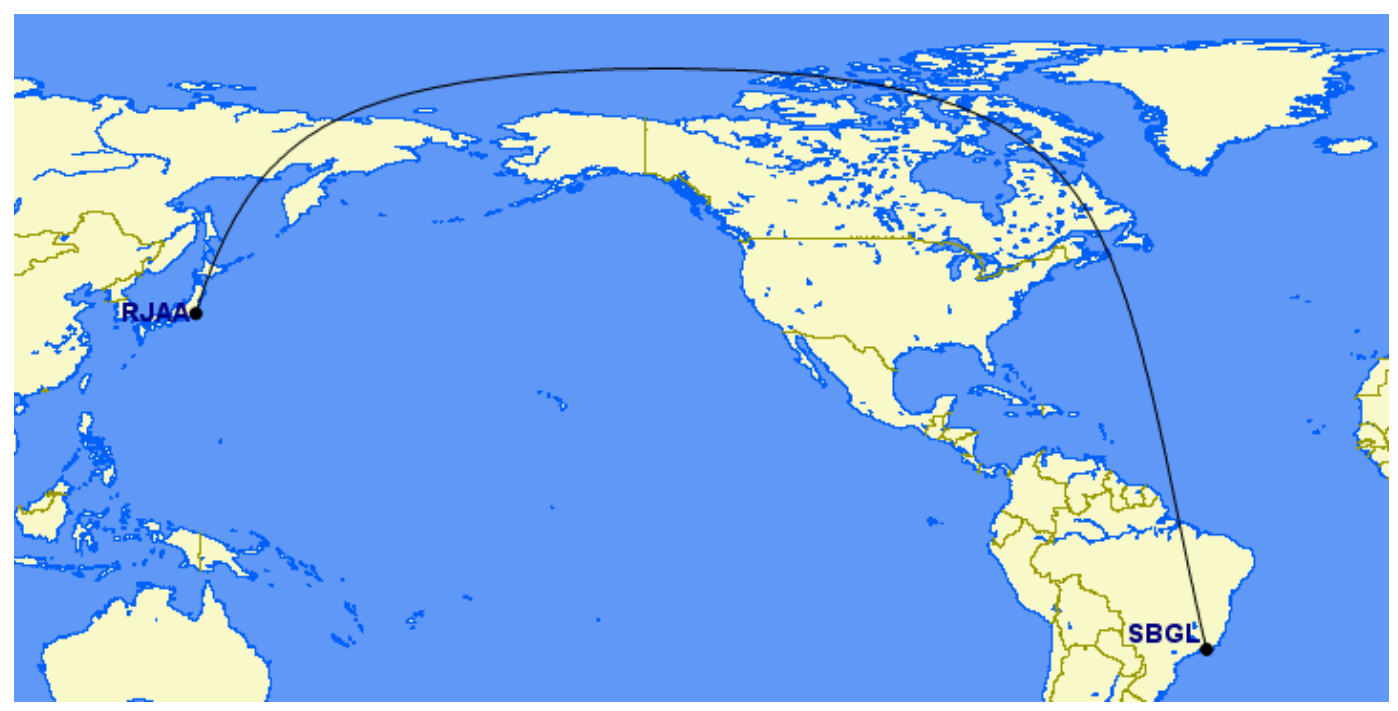

Figure 7. GC (geodesic) route SBGL to RJAA (GIG to NRT) on conformal cylindrical Mercator chart. Courtesy of GC Mapper. Maps generated by the Great Circle Mapper (www.gcmap.com) - copyright @ Karl L. Swartz.

The last route we specifically discuss is also the longest flight of all identified here between the existing major airports. In Figures 9 and 10 the route between Quito (Ecuador) and Kuala Lumpur (Malaysia) or SEQM to WMKK (UIO to KUL) are shown respectively on the Polar Orthographic and the Mercator charts. Both cities lie almost exactly on Equator and are very close to be antipodal. Our navigation calculator returned the value of 10,667.53 NM for GC distance flying average altitude of $36,000 \mathrm{ft}$ with outbound initial heading of $358.510^{\circ}$ and inbound destination heading into WMKK on $181.492^{\circ}$ heading. The vertex calculated is at Lat/Long $+88.5099^{\circ} /-169.850^{\circ}$ and which is only about 90 NM from the NP. Detailed listing of the route is given in Appendix F.

The rhumb-line calculations returned the distance of $10,819.16 \mathrm{NM}$ at a constant heading of $270.91^{\circ}$. The GC Mapper returned the value of 10,644 NM for the shortest (geodesic) distance over oblate Earth. However, that is surface distance and if we add about $18 \mathrm{NM}$ for additional air distance we arrive at 10,662 
NM which is within 6 NM $(0.052 \%)$ of our calculations. GC Mapper returned a value of $358.8^{\circ}$ for outbound course from departure point SEQM, which is within 18 angular minutes of our calculations. The Onboardintelligence.com calculator returned the value of 10,644.04 $\mathrm{NM}$ for the Orthodrome and 10,812.68 at 270.9 for the Loxodrome. The GC departure course calculated is $360^{\circ}$ and destination inbound course is $181.2^{\circ}$. Vertex is at $\mathrm{N} 88^{\circ} 47^{\prime} .3$ and $\mathrm{W} 168^{\circ} 21^{\prime} .3$. Due to actual oblateness of the Earth, the shortest distance between two locations close to Equator and on opposite meridians very likely will go over the NP or the SP.
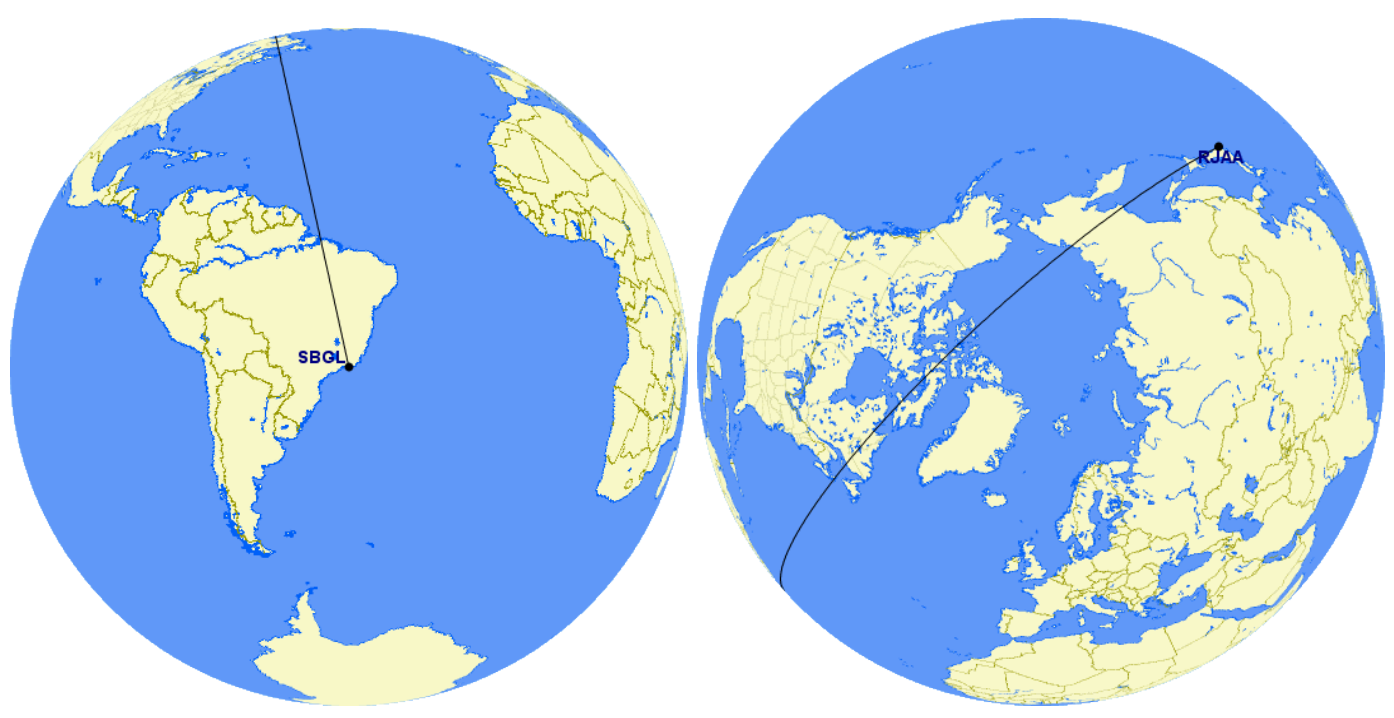

Figure 8. Geodesic route SBGL to RJAA (GIG to NRT) on Polar Orthographic chart. Courtesy of GC Mapper. Maps generated by the Great Circle Mapper (www.gcmap.com) - copyright (c) Karl L. Swartz.

A familiar example of long-range route within continental US is flight from KJFK (New York) to KLAX (Los Angeles). Our calculations have been verified against Phillips' (2004). A GC route at FL360 is 2,148.87 NM long with the outbound course of $273.858^{\circ}$ and the KLAX inbound of $245.892^{\circ}$. The vertex is reached shortly after departing KJFK westbound. The rhumb-line distance is 2,169.77 $\mathrm{NM}$ at constant $259.324^{\circ} \mathrm{TC}$ and just about $21 \mathrm{NM}$ longer than the Orthodrome. Many examples of long-range flights (see Table 2) do not show very large difference between the GC and rhumb-line distances - often less than 5\% (except MMMX-VCBI). In fact, the difference is largest when flying between two points of similar mid-latitudes. For example, KMSP (Table 1) and Urumchi (Ürümqi) Diwopu International Airport in China, Xinjiang/Uyghur province (ICAO: ZWWW, IATA: URC) with latitude $+43.908^{\circ}$ and longitude of $+87.475^{\circ}$, are practically located on a meridian and its anti-meridian (longitude change of 
$179.3^{\circ}$ ). Both are located around the $44^{\text {th }}$ parallel (like Sarajevo/LQSA). In fact the central angle (about $91.21^{\circ}$ ) of the two airports lies in an osculating plane slicing almost exactly over the NP. The orthodrome-arc length on the surface of the spherical Earth is $10,142.1 \mathrm{~km}(5,476.3 \mathrm{NM})$, while the Loxodrome will almost follow $44^{\text {th }}$ parallel. The Loxodrome is about $14,244.6 \mathrm{~km}(7,691.5 \mathrm{NM})$ which is about $40.5 \%$ longer than the GC-arc. Rhumb-line flying will imply following straight East or West TC while the Orthodrome departure from KMSP is almost on a straight North TC. Flying at FL360 will add about 10 NM. Flight LQSA to KMSP shows $9.1 \%$ difference between the GC and the rhumb-line.

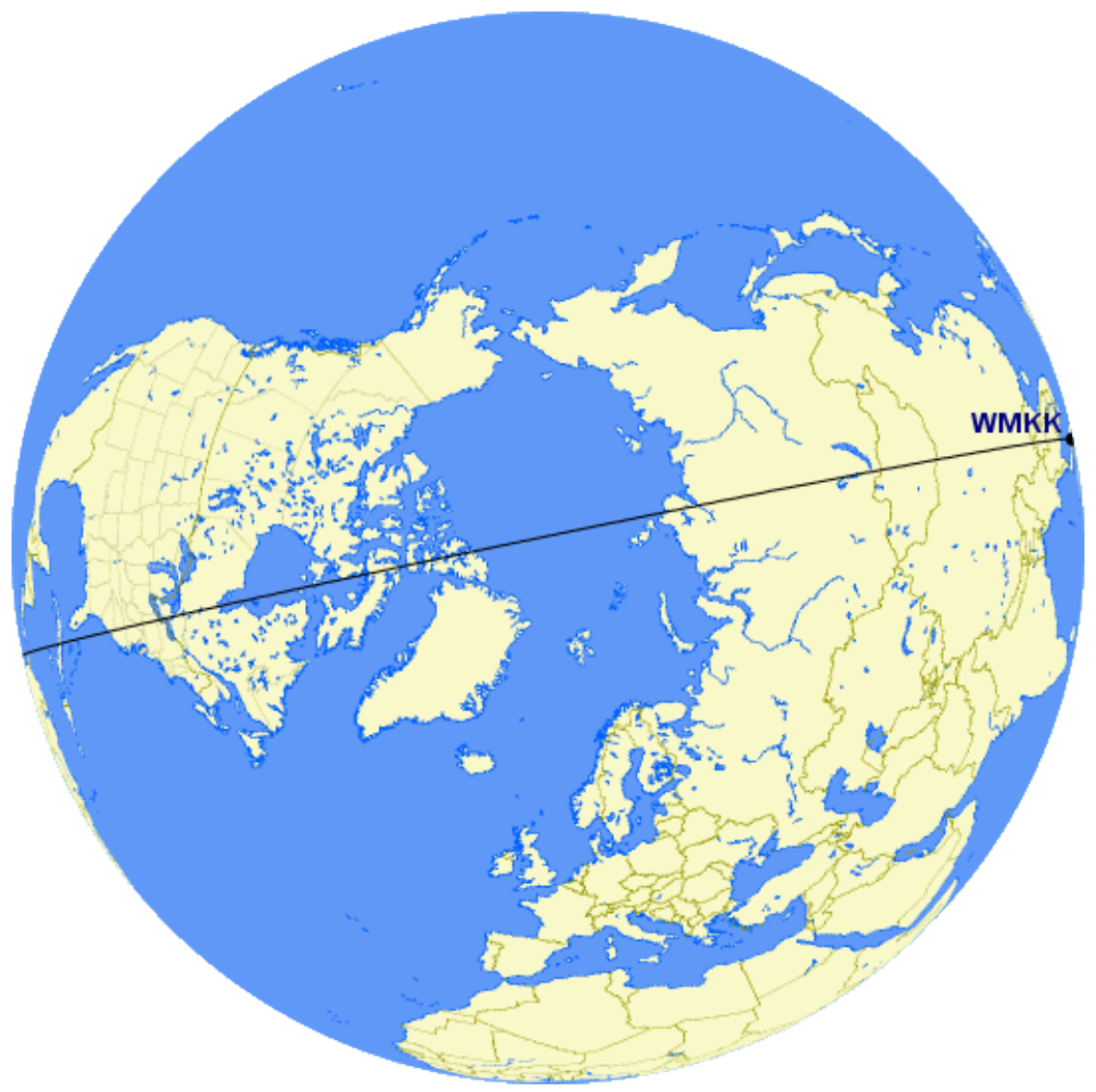

Figure 9. Geodesic route SEQM to WMKK (UIO to KUL) on Polar Orthographic chart. Courtesy of GC Mapper. Maps generated by the Great Circle Mapper (www.gcmap.com) - copyright (C) Karl L. Swartz.

It must be said that GC distance calculations are very robust as they involve cosines which is an even function. On the other hand course calculations are fragile and considerable effort was made to make spherical-angles 
transformation into Earth's coordinates accurate under all conditions. All test routes evaluated at MSL showed excellent agreement with the available terrestrial spheroid geodesic calculators. However, these publically-available calculators are black-boxes with no insight to inner workings, plus they do not offer the ability to include altitude corrections. Our GC and rhumb-line courses and angles computations also showed excellent agreement with other calculators. Hence, we gained confidence in our navigation programs which can calculate almost any route on Earth with sufficiently high accuracy apart from the true antipodal distances for which the problem becomes undetermined on the spherical Earth.

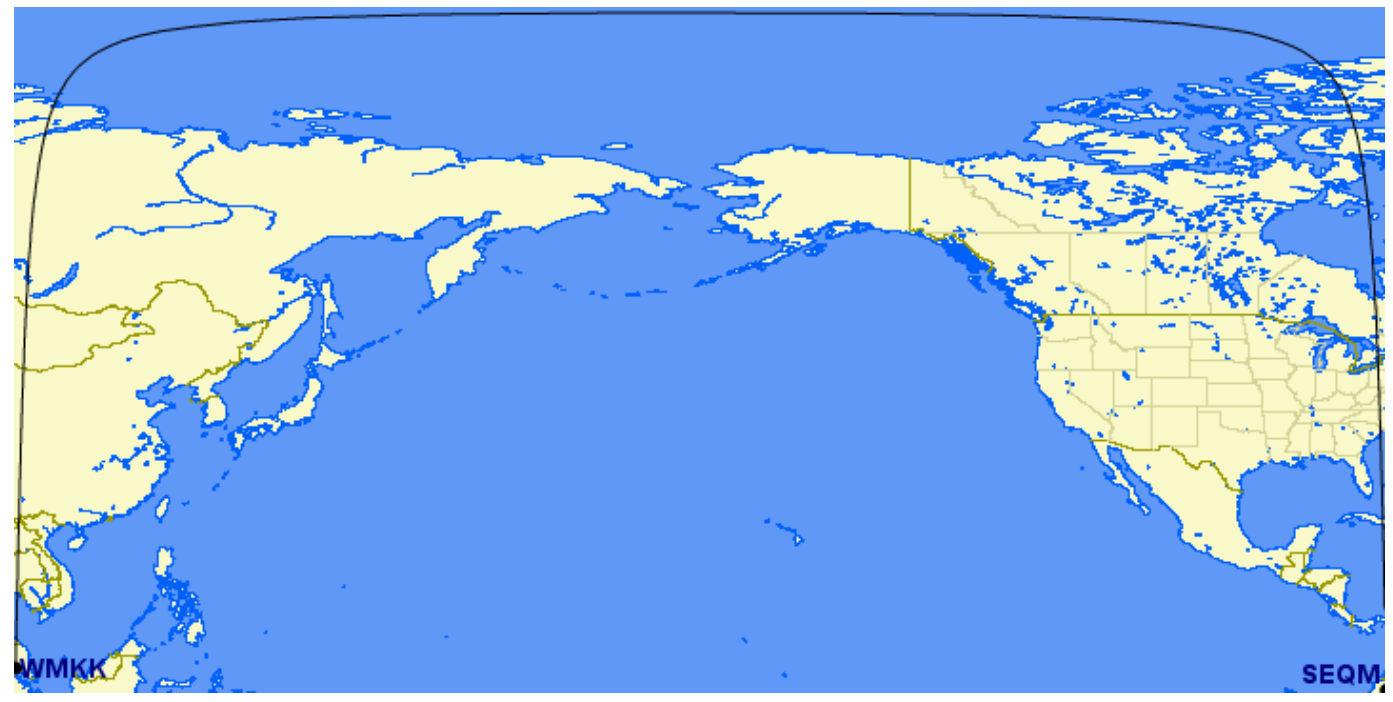

Figure 10. Geodesic route SEQM to WMKK (UIO to KUL) on conformal cylindrical Mercator chart. Courtesy of GC Mapper. Maps generated by the Great Circle Mapper (www.gcmap.com) - copyright (C Karl L. Swartz.

A common misconception when considering GC-route flying is that somehow since it is a curve on Mercator chart it should be approximated by straight line (secant) segments. As a matter of fact as long as the weight vector remains in the osculating plane no change of flight course is required for nonrotating planet. In the absence of wind and GC trajectories, an aircraft only needs to assume the correct initial heading and continue with no rolling and/or yawing motion. GC arc is a geodesic with the projection on a tangential plane being a straight line and thus shortest distance. The geodesic curvature is zero. The effect of Coriolis force exists for rotating planet and will be discussed in a future contribution. It is in fact the loxodromic route that requires yawing motion to maintain constant heading on spherical Earth (see Figure C2). Loxodrome ends up being an infinite logarithmic spiral of finite length that winds around the pole. 
We are currently implementing IDL mapping capabilities using various Earth projections. An older licensed RSI's IDL v.5.6 (2002) is being currently used. Generally, IDL (newest v.8.6 by Harris Geospatial) has very rich and powerful mapping and plotting capabilities and can work as standalone program or as a graphic interface to modern Fortran, Matlab, and Basic programs. Calculations of segment PNR and PET for given aircraft model in arbitrary wind conditions (Daidzic, 2016a) are easily implemented. Also all-engines-inoperative gliding performance with arbitrary wind can be added for routes. Some of the calculated routes from Table 2 can be easily verified manually using the basic Orthodrome and Loxodrome distance formulas given in Equations (2-4). More improvements and testing will be conducted in the future on our AARNAV ${ }^{\mathrm{TM}}$ airnavigation calculators adding more graphic features and capabilities and enhancing performance. GC and rhumb-line waypoints can be calculated or for equidistant arc segments or for constant longitude increments (Appendix F). Loxodrome, GE, and true geodesic calculations on reference WGS-84 ellipsoid will be added to GC and loxodrome calculations on spherical Earth.

\section{Short lines on spherical Earth}

First we test polar short-distance calculators based on the planar projections. As an example we took two points on Antarctica close to the SP point 1 has coordinates $\mathrm{S}^{\circ} 0^{\circ} 20^{\prime} 30.5000^{\prime \prime}$ and E100 30'40.3456", while point 2 has coordinates ${\mathrm{S} 85^{\circ}}^{\circ} 10^{\prime} 44.7575^{\prime \prime}$ and E150 $45^{\prime}$ '20.0000". The exact GC inverse-cosine and inverse haversine formulas both returned the value of 453.43222 NM $(839.75647 \mathrm{~km})$, while the Law-of-Cosine flat-Earth approximation (Equation D2) returned the value of 453.1708446 NM. The difference is less than $1,590 \mathrm{ft}$ or about $484 \mathrm{~m}(0.058 \%$ error $)$, which is excellent accuracy for distance of about $840 \mathrm{~km}$.

Several right-angle very short-distance calculations (Equation D4) and comparison with the true GC formulas were also conducted. Mankato regional airport (KMKT) has coordinates (N44 13 ' 22.0000" and W093 ${ }^{\circ} 55^{\prime}$ 09.5000"). A close by Le Sueur (12Y) airport is almost straight true North (TN) with the coordinates ( $\mathrm{N}_{4}^{\circ}{ }^{\circ} 26^{\prime} 27.1971$ " and $\mathrm{W} 093^{\circ}$ 54' 57.0502"). Our GC calculators using inverse-cosine and inverse haversine formulas (Equations 2 and 3) both returned distance of $13.118826 \mathrm{NM}$, while the planar approximation (Equation D4) returned the value of $13.09708846 \mathrm{NM}$, which is only about $132.16 \mathrm{ft}(40.28$ $\mathrm{m})$ difference. In another example, for two points close to equator with the change of latitude of 10 arc-minutes (about $10 \mathrm{NM}$ ) symmetric across the equator and the difference of longitude of 10 arc-minutes, the exact formulas return equal distance 
of $14.17603 \mathrm{NM}(26.254 \mathrm{~km})$, while the flat-Earth approximation returns the value of $14.15167 \mathrm{NM}$, which is only $148.08 \mathrm{ft}$ or less than $0.17 \%$ error.

On the other hand if we use KMKT as departure airport again and utilize the flat-Earth approximation to calculate distance to a nearby Waseca airport (KACQ), which is just about 9 arc-minutes to the south and 22 arc-minutes to the East at about $44^{\text {th }}$ parallel, we obtain "straight" distance of $23.753 \mathrm{NM}$. This is 5+ NM (actually 33,867 ft) longer than the exact inverse-cosine or haversine GC formula delivering about $18.183 \mathrm{NM}$ and thus unacceptably inaccurate. The shortline distance formula given with Equation (D4) is acceptable only up to about 15 NM $(28 \mathrm{~km})$ in equatorial and lower mid-latitudes. Calculations were performed utilizing 32 and 64-bit floating point arithmetic with MS Excel, True Basic v. 5.5 and v.6, Fortran 90/95/2003/2008, and Matlab R2015a (8.5) high-level computerlanguage codes. We anticipate that a more user-friendly and thoroughly tested program version will be offered in the future for free to all users in public domain.

\section{Conclusions}

Global range air navigation implies flying non-stop from any airport to any other airport on Earth. That requires airplanes with the operational air range of at least 12,500 NM. Air transportation economy requires shortest distance flights which in the case of spherical Earth are Orthodrome arcs. Rhumb-line navigation has no practical application in long-range flights but has been used as comparison for historical reasons. Great Circle routes between many major international airports have been calculated and waypoints presented for both GC and rhumb-line routes. Many future global-range flights may be prohibited due to polar crossings and/or long flights over open water with not many alternate landing sites available. Additionally, we summarized short-lines navigation theory with particular emphasis on Polar Regions and very short distances elsewhere on the Earth. Working equations and algorithms have been coded into several highlevel programming languages. Considerable testing of programs have been conducted and compared with the publically-available geodesic computations over the surface of the terrestrial reference ellipsoid. Distance computations usually were less than $0.3 \%$ in error, while the angles and courses were mostly within few angular minutes. Accurate database of about 50 major international airports from every corner of the world has been constructed and used in testing and route validation. Further development will include computations of gliding distances from any altitude under arbitrary winds depending on the type of aircraft and the calculations of PET and PNR for every segment of the route and arbitrary wind conditions. A user-friendly machine-independent program version for global navigation with many flight planning features will be posted to public domain. 


\section{Author Bios}

Dr. Nihad E. Daidzic is president of AAR Aerospace Consulting, L.L.C. He is also a full professor of Aviation, adjunct professor of Mechanical Engineering, and research graduate faculty at Minnesota State University. His Ph.D. is in fluid mechanics and Sc.D. in mechanical engineering. He was formerly a staff scientist at the National Center for Microgravity Research and the National Center for Space Exploration and Research at NASA Glenn Research Center in Cleveland, $\mathrm{OH}$. He also held various faculty appointments at Vanderbilt University, University of Kansas, and Kent State University. His current research interest is in theoretical, experimental, and computational fluid dynamics, micro- and nanofluidics, aircraft stability, control, and performance, mechanics of flight, piloting techniques, and aerospace propulsion. Dr. Daidzic is ATP and "Gold Seal" CFII/MEI/CFIG with flight experience in airplanes, helicopters, and gliders. 


\section{References}

Abramowitz, M., Stegun, I. A. (1984). Handbook of mathematical functions (abridged edition). Frankfurt (am Main), Germany: Verlag Harri Deutsch.

Aleksandrov, A. D., Kolmogorov, A. N., \& Lavrent'ev, M. A. (1999). Mathematics: Its content, methods and meaning (3 volumes translation from Russian). Mineola, NY: Dover.

Alexander, J. (2004). Loxodromes: A rhumb way to go, Mathematics Magazine, 77(5), 349-356. DOI: 10.2307/3219199

Ayres, F., Jr., \& Mendelson,E. (2009). Calculus (5th ed.). New York, NY: McGraw-Hill.

Bate, R.R., Mueller, D. D., \& White J. E. (1971). Fundamentals of astrodynamics. New York, NY: Dover.

Bennett, G. G. (1996). Practical rhumb line calculations on a spheroid. Journal of Navigation, 49(2), 112-119. DOI: 10.1017/s0373463300013151

Bomford, G. (1983). Geodesy (reprinted 4th ed.). New York, NY: Oxford University Press.

Bowditch, N. (2002). The American Practical Navigator (2002 bicentennial ed.). Bethesda, MD: National Imagery and Mapping Agency (NIMA).

Bowring, B. R. (1984). The direct and inverse solutions for the great elliptic line on the reference ellipsoid. Bulletin Géodésique 58(1), 101-108. DOI: 10.1007/BF02521760

Bradley, A. D. (1942). Mathematics of air and marine navigation with tables. New York, NY: American Book Company.

Bronstein, I. N., \& Semendjajew, K. A. (1989). Taschenbuch der Mathematik (24. Auflage). Frankfurt/Main, Germany: Verlag Harri Deutsch.

Byrd, P. F., \& Friedman, M. D. (1954). Handbook of elliptic integrals for engineers and physicists. Berlin, Germany: Springer Verlag. 
Daidzic, N. E. (2014). Achieving global range in future subsonic and supersonic airplanes. International Journal of Aviation, Aeronautics, and Aerospace (IJAAA), 1(4), 1-29. DOI: 10.15394/ijaaa.2014.1038

Daidzic, N. E. (2016a). General solution of the wind triangle problem and the critical tailwind angle, The International Journal of Aviation Sciences (IJAS), 1(1), 57-93.

Daidzic, N. E. (2016b). Estimation of performance airspeeds for high-bypass turbofans equipped transport-category airplanes. Journal of Aviation Technology and Engineering (JATE), 5(2), pp. 27-50. DOI: 10.7771/21596670.1122

Danby, J. M. A. (1962). Fundamentals of celestial mechanics. New York, NY: MacMillan.

De Florio, F. (2016). Airworthiness: An introduction to aircraft certification and operations (3rd ed.). Oxford, UK: Butterworth-Heinemann.

De Remer, D., \& McLean, D. W. (1998). Global navigation for pilots (2nd ed.). Newcastle, WA: Aviation Supplies and Academics, Inc.

Dym, C. L., Shames, I. H. (2013). Solid mechanics: A variational approach (Augmented edition). New York, NY: Springer. DOI: 10.1007/978-14614-6034-3

Dwight, H. B. (1961). Tables of integrals and other mathematical data (4th ed.). New York, NY: Macmillan.

Fitzpatrick, R. (2012). An introduction to celestial mechanics. Cambridge, UK: Cambridge University Press.

Fox, C. (1987). An introduction to the calculus of variation. Mineola, NY: Dover.

Goetz, A. (1970). Introduction to differential geometry. Reading, MA: AddisonWesley.

Greenwood, D. T. (1987). Classical dynamics. New York, NY: Dover.

Hall, J. E. (1968). Analytic geometry. Belmont, CA: Brooks/Cole Publishing Co. 
Jahnke, E., \& Emde, F. (1945). Tables of functions: With formulae and curves. New York, NY: Dover.

Jekeli, C. (2012). Geometric reference systems in geodesy. Columbus, OH: State University.

Jeppesen. (2007). General navigation. (JAA ATPL Training, Edition 2, Atlantic Flight Training, Ltd., Sanderson Training products), Neu-Isenburg, Germany: Author.

Karney, C. F. F. (2013). Algorithms for geodesics. J. Geod., 87, pp. 43-55. DOI: $10.1007 / \mathrm{s} 00190-012-0578-\mathrm{z}$

Kos, S., Vranić D., \& Zec, D. (1999). Differential equation of a loxodrome on a sphere. Journal of Navigation, 52(3), 418-420. DOI: $10.1017 / \mathrm{s} 0373463399008395$

Krakiwsky, E. J., \& Thomson, D. B. (1974). Geodetic position computations. Dept. of Geodesy and Geomatics Engineering, Lecture Notes (39), Fredericton, N.B., Canada: University of New Brunswick.

Kreyszig, E. (1964). Differential geometry (revised ed.). Toronto, Canada; University of Toronto Press.

Lanczos, C. (1986). The variational principles of mechanics (4th ed.). Mineola, NY: Dover.

Lass, H. (2009). Elements of pure and applied mathematics. Mineola, NY: Dover.

Lipschutz, M. M. (1969). Differential geometry. New York, NY: McGraw-Hill.

Lowrie, W. (2007). Fundamentals of geophysics (2nd ed.). Cambridge, UK: Cambridge University Press.

McIntyre, D. H. (2000). Using great circles to understand motion on rotating sphere. Am. J. Phys., 68(12), 1097-1105, DOI: 10.1119/1.1286858

Miele, A. (2016). Flight mechanics: Theory of flight paths. Mineola, NY: Dover. 
Nielsen, K. L., \& Vanlonkhuyzen, J. H. (1954). Plane and spherical trigonometry. New York, NY: Barnes \& Noble.

Olza, A., Taillard, F., Vautravers, E., \& Diethelm, J. C. (1974). Tables numériques et formulaires. Lausanne, Switzerland: Spes S.A.

Oprea, J. (2007). Differential geometry and its applications (2nd ed.). Washington, DC: The Mathematical Association of America.

Phillips, W. F. (2004). Mechanics of flight. New York, NY: John Wiley \& Sons.

Rapp, R. H. (1991). Geometric geodesy: Part I. Columbus, OH: State University.

Rapp, R. H. (1993). Geometric geodesy: Part II. Columbus, OH: State University.

Rollins, C. M. (2010). An integral for geodesic length. Survey Review, 42(315), 20-26. DOI: 10.1179/003962609X451663

Smith, D. R. (1998). Variational methods in optimization. Mineola, NY: Dover.

Spiegel, M. R., \& Liu, J. (1999). Mathematical handbook of formulas and tables (2nd ed.). New York, NY: McGraw-Hill.

Sinnott, R. W. (1984). Virtues of the haversine. Sky and Telescope. 68(2), 159.

Sjöberg, L. E. (2012). Solutions to the direct and inverse navigation problems on the great ellipse. Journal of Geodetic Science, 2(3), 200-205. DOI: $10.2478 / \mathrm{v} 1015601100409$

Struik, D. J. (1987). A concise history of mathematics (4th). Mineola, NY: Dover.

Struik, D. J. (1988). Lectures on classical differential geometry (2nd ed.). Mineola, NY: Dover.

Tewari, A. (2007). Atmospheric and space flight dynamics: Modeling and simulation with Matlab ${ }^{\circledR}$ and Simulink ${ }^{\circledR}$. Boston, MN: Birkhäuser.

Tikhonov, A. N., \& Samarskii, A. A. (1990). Equations of mathematical physics. Mineola, NY: Dover.

Todhunter (1886). Spherical trigonometry (5th ed.). London, UK: MacMillan. 
Tooley, M., \& Wyatt, D. (2007). Aircraft communications and navigation systems: Principles, maintenance and operation. London, UK: Taylor \& Francis.

Torge, W. (2001). Geodesy ( ${ }^{\text {rd }}$ ed.). Berlin, Germany: Walter de Gruyter, GmbH.

Tseng, W.-K., \& Lee, H.-S. (2010). Navigation on a great ellipse. Journal of marine science and technology, 18(3), 369-375.

Underdown, R. B., \& Palmer, T. (2001). Navigation: ground studies for pilots. 6th ed. Oxford, UK: Blackwell Science, Ltd.

US Department of Transportation, Federal Aviation Administration. (2008). Extended operations (ETOPS and Polar operations) (Advisory Circular AC 120-42B). Washington, DC: Author.

Vaníček, P., \& Krakiwsky, E. (1986). Geodesy: The concepts (2nd ed.). New York, NY: North-Holland.

Vincenty, T. (1975). Direct and inverse solutions of geodesics on the ellipsoid with application of nested equations. Survey Review, 23(176), 88-93. DOI: $10.1179 /$ sre.1975.23.176.88

Weber, H. J., \& Arfken, G. B. (2004). Essential mathematical methods for physicists. Amsterdam, the Netherlands: Elsevier.

Weintrit, A., \& Kopacz, P. (2011). A novel approach to loxodrome (rhumb line), orthodrome (great circle) and geodesic line in ECDIS and navigation in general, Int. J. Marine Nav. and Safety Sea Transp., 5(4), 507-517. DOI: 10.1201/b11344-21.

Widder, D. V. (1989). Advanced calculus (2nd ed.). New York, NY: Dover.

Williams, E. (2011). Aviation formulary V1.46. Retrieved from http://williams.best.vwh.net/avform.htm.

Williams, J. E. D. (1950). Loxodromic distances on the terrestrial spheroid. Journal of Navigation, 3(2), pp. 133-140. DOI: $10.1017 / \mathrm{s} 0373463300045549$ 
International Journal of Aviation, Aeronautics, and Aerospace, Vol. 4 [2017], Iss. 1, Art. 2

Williams, R. (1996). The great ellipse on the surface of the spheroid. Journal of Navigation, 49(2), 229-234. DOI: 10.1017/s0373463300013333

Wolper, J. S. (2001). Understanding mathematics for aircraft navigation. New York, NY; McGraw-Hill.

Wrede, R. C. (1972). Introduction to vector and tensor analysis. New York, NY: Dover. 


\section{Appendix A}

Fundamental geometrical and topological properties of spheres

In this section fundamental properties of spheres will be given. Basic familiarity with classical differential geometry and topology (Goetz, 1970; Kreyszig, 1964; Lipschutz, 1969; Oprea, 2007; Struik 1988; Widder, 1989; Wrede, 1972) is required. Euclidian geometry is assumed. A spherical coordinate system used in geodesy and terrestrial (air, maritime, etc.) navigation is somewhat different from the conventional used in mathematical physics (Tikhonov and Samarskii, 1990). For the homogeneous smooth sphere of constant radius for which the center of mass (barycenter) is in the geocenter, we have:

$$
\begin{array}{lrr}
x=R \cdot \cos \phi \cdot \cos \lambda & y=R \cdot \cos \phi \cdot \sin \lambda & z=R \cdot \sin \phi \\
-\pi / 2 \leq \phi \leq \pi / 2 & -\pi \leq \lambda \leq \pi
\end{array}
$$

We designated $\phi$ as latitude (geocentric and geodetic) measured from equatorial plane, and $\lambda$ is latitude. Spherical coordinates can be represented inversely in terms of Cartesian coordinates:

$$
\begin{aligned}
& R=\sqrt{x^{2}+y^{2}+z^{2}} \quad \phi=\sin ^{-1}(z / R)=\tan ^{-1}\left(z /\left(x^{2}+y^{2}\right)\right) \quad \lambda=\tan ^{-1}(y / x) \\
& -R \leq x \leq R \quad-R \leq y \leq R \quad-R \leq z \leq R
\end{aligned}
$$

The first fundamental form of differential geometry specifies positive definite invariant or arc length of the surface given parametrically as $x_{i}=x_{i}(u, v)=x_{i}(\phi, \lambda)($ Equation A1):

$$
\begin{aligned}
& I=d s^{2}=d \mathbf{x} \cdot d \mathbf{x}=d x_{i} \cdot d x_{i}=\left(\mathbf{x}_{u} d u+\mathbf{x}_{v} d v\right) \cdot\left(\mathbf{x}_{u} d u+\mathbf{x}_{v} d v\right)= \\
& =E d u^{2}+2 F d u d v+G d v^{2} \geq 0
\end{aligned}
$$

where,

$$
E=\frac{\partial x_{i}}{\partial u} \cdot \frac{\partial x_{i}}{\partial u}=\mathbf{x}_{u} \cdot \mathbf{x}_{u} \quad F=\frac{\partial x_{i}}{\partial u} \cdot \frac{\partial x_{i}}{\partial v}=\mathbf{x}_{u} \cdot \mathbf{x}_{v} \quad G=\frac{\partial x_{i}}{\partial v} \cdot \frac{\partial x_{i}}{\partial v}=\mathbf{x}_{v} \cdot \mathbf{x}_{v}
$$

For a sphere given in spherical coordinates $u=\phi$ and $v=\lambda$ (Equation A1), we obtain: 
$\mathbf{x}_{u}=\mathbf{x}_{\phi}=(-R \cdot \sin \phi \cdot \cos \lambda) \cdot \mathbf{i}+(-R \cdot \sin \phi \cdot \sin \lambda) \cdot \mathbf{j}+(R \cdot \cos \phi) \cdot \mathbf{k}$

$\mathbf{x}_{v}=\mathbf{x}_{\lambda}=(R \cdot \cos \phi \cdot \sin \lambda) \cdot \mathbf{i}+(-R \cdot \cos \phi \cdot \cos \lambda) \cdot \mathbf{j}+(0) \cdot \mathbf{k}$

Where,

$$
E=\mathbf{x}_{\phi} \cdot \mathbf{x}_{\phi}=R^{2} \quad F=\mathbf{x}_{\phi} \cdot \mathbf{x}_{\lambda}=0 \quad G=\mathbf{x}_{\lambda} \cdot \mathbf{x}_{\lambda}=R^{2} \cos ^{2} \phi
$$

For a sphere we thus have:

$$
d s^{2}=|d \mathbf{x}| \cdot|d \mathbf{x}|=R^{2}\left(d \phi^{2}+\cos ^{2} \phi d \lambda^{2}\right)
$$

A vector product of parametric tangent lines is:

$$
\begin{aligned}
& \mathbf{x}_{\phi} \times \mathbf{x}_{\lambda}=\left|\begin{array}{ccc}
\mathbf{i} & \mathbf{j} & \mathbf{k} \\
x_{\phi} & y_{\phi} & z_{\phi} \\
x_{\lambda} & y_{\lambda} & z_{\lambda}
\end{array}\right|= \\
& =\left[\left(y_{\phi} z_{\lambda}-y_{\lambda} z_{\phi}\right) \cdot \mathbf{i}-\left(x_{\phi} z_{\lambda}-x_{\lambda} z_{\phi}\right) \cdot \mathbf{j}+\left(x_{\phi} y_{\lambda}-x_{\lambda} y_{\phi}\right) \cdot \mathbf{k}\right]= \\
& =\left(-R^{2} \cdot \cos ^{2} \phi \cdot \cos \lambda\right) \cdot \mathbf{i}+\left(-R^{2} \cdot \cos ^{2} \cdot \phi \sin \lambda\right) \cdot \mathbf{j}+\left(-R^{2} \cdot \sin \phi \cdot \cos \phi\right) \cdot \mathbf{k}
\end{aligned}
$$

An important property for a sphere (Struik, 1988) that is easily derived from vector calculus and will be often used is:

$$
\begin{aligned}
& \left|\mathbf{x}_{\phi} \times \mathbf{x}_{\lambda}\right|^{2}=\left(\mathbf{x}_{\phi} \times \mathbf{x}_{\lambda}\right) \cdot\left(\mathbf{x}_{\phi} \times \mathbf{x}_{\lambda}\right)=\left(\mathbf{x}_{\phi} \cdot \mathbf{x}_{\phi}\right) \cdot\left(\mathbf{x}_{\lambda} \cdot \mathbf{x}_{\lambda}\right)-\left(\mathbf{x}_{\phi} \cdot \mathbf{x}_{\lambda}\right)^{2}= \\
& \left|\mathbf{x}_{\phi} \times \mathbf{x}_{\lambda}\right|=\sqrt{E G-F^{2}}=R^{2} \cos \phi \geq 0 \quad \phi \in\left[-\frac{\pi}{2},+\frac{\pi}{2}\right]
\end{aligned}
$$

If the curvilinear surface coordinates are further a function of a single parameter, i.e., $x_{i}=x_{i}(u(t), v(t))$, the arc length is (Goetz, 1970; Kreyszig, 1964; Lipschutz, 1969; Oprea, 2007; Struik 1988; Widder, 1989):

$$
s=\int_{a}^{b} \sqrt{I} d t=\int_{a}^{b}\left[E\left(\frac{d u}{d t}\right)^{2}+2 F\left(\frac{d u}{d t} \frac{d v}{d t}\right)+G\left(\frac{d v}{d t}\right)^{2}\right]^{1 / 2} d t \quad t \in[a, b]
$$

Or we can write for a sphere: 
$s=\int_{\phi_{1}}^{\phi_{2}}\left[E+G\left(\frac{d \lambda}{d \phi}\right)^{2}\right]^{1 / 2} d \phi=\int_{\lambda_{1}}^{\lambda_{2}}\left[E\left(\frac{d \phi}{d \lambda}\right)^{2}+G\right]^{1 / 2} d \lambda$

For a curve coinciding with a meridian (line of longitude) and measuring from SP to NP we have $d \lambda=0$, and:

$S=\int_{-\pi / 2}^{+\pi / 2} \sqrt{E} d \phi=R \int_{-\pi / 2}^{+\pi / 2} d \phi=R \cdot \pi$

For an arbitrary line of latitude $\left(\phi=\phi_{0}, d \phi=0\right)$, we obtain:

$s=\int_{-\pi}^{+\pi} \sqrt{G} d \lambda=R \int_{-\pi}^{+\pi} \cos \phi_{0} d \lambda=2 \pi \cdot R \cos \phi_{0}$

Small circles will have progressively shorter arcs of length until respective poles where this becomes zero. The NP and the SP are the singular points on the sphere (Struik, 1988).

The local angle between vectors $d \mathbf{x}$ and $\delta \mathbf{x}$ parallel to a tangent plane at an arbitrary point on sphere is (Lipschutz, 1969; Struik 1988):

$$
\begin{aligned}
& \cos \alpha=\frac{d x_{i} \cdot \delta x_{i}}{\left|d x_{i}\right| \cdot\left|\delta x_{i}\right|}=\frac{d \mathbf{x} \cdot \delta \mathbf{x}}{|d \mathbf{x}| \cdot|\delta \mathbf{x}|}=\frac{\left(\mathbf{x}_{u} d u+\mathbf{x}_{v} d v\right) \cdot\left(\mathbf{x}_{u} \delta u+\mathbf{x}_{v} \delta v\right)}{\left|\mathbf{x}_{u} d u+\mathbf{x}_{v} d v\right| \cdot\left|\mathbf{x}_{u} \delta u+\mathbf{x}_{v} \delta v\right|}= \\
& =\frac{E d u \delta u+F(d u \delta v+d v \delta u)+G d v \delta v}{\left[E d u^{2}+2 F d u d v+G d v^{2}\right]^{1 / 2} \cdot\left[E \delta u^{2}+2 F \delta u \delta v+G \delta v^{2}\right]^{1 / 2}}= \\
& =E \frac{d u}{d s} \frac{\delta u}{\delta s}+F\left(\frac{d u}{d s} \frac{\delta v}{\delta s}+\frac{d v}{d s} \frac{\delta u}{\delta s}\right)+G \frac{d v}{d s} \frac{\delta v}{\delta s}
\end{aligned}
$$

For the tangent lines on the parametric curves (graticule - network of lines of latitudes and longitudes) the above expression implies that the scalar product must be zero as they are orthogonal as required by chart conformality. Indeed, using Equation (A4), we obtain the condition of orthogonality of parametric lines:

$\mathbf{x}_{\phi} \cdot \mathbf{x}_{\lambda}=0 \Rightarrow E d \phi \delta \phi+F(d \phi \delta \lambda+d \lambda \delta \phi)+G d \lambda \delta \lambda=0$ 
The angle between the parametric lines ( $u=$ const., $d u=0, d v$ arbitrary) and ( $v=$ const, $\delta v=0, \delta u$ arbitrary ) and using Equation (A12), results in:

$$
\cos \theta=\frac{F d v \delta u}{\sqrt{G d v^{2}} \cdot \sqrt{E \delta u^{2}}}=\frac{F}{\sqrt{E G}} \quad \sin \theta=\frac{\sqrt{E G-F^{2}}}{\sqrt{E G}}
$$

Clearly, the coefficient $F$ must be zero for the cosine angle to be zero and sine to be one resulting in the right angle solution. The unit vector normal on the parametric surface $\mathbf{x}=\mathbf{x}(u, v)$ at an arbitrary point using Equations (A8) and (A9) is (Lipschutz, 1969; Struik 1988; Widder, 1989):

$$
\mathbf{N}=\frac{\mathbf{x}_{u} \times \mathbf{x}_{v}}{\left|\mathbf{x}_{u} \times \mathbf{x}_{v}\right|}=\frac{\mathbf{x}_{\phi} \times \mathbf{x}_{\lambda}}{\left|\mathbf{x}_{\phi} \times \mathbf{x}_{\lambda}\right|}=\frac{1}{\sqrt{E G-F^{2}}} \cdot\left|\begin{array}{ccc}
\mathbf{i} & \mathbf{j} & \mathbf{k} \\
x_{\phi} & y_{\phi} & z_{\phi} \\
x_{\lambda} & y_{\lambda} & z_{\lambda}
\end{array}\right|
$$

By substituting partial derivatives for a sphere, the surface unit normal becomes:

$$
\mathbf{N}=(-\cos \phi \cdot \cos \lambda,-\cos \phi \cdot \sin \lambda,-\sin \phi)
$$

The surface normal thus points toward the center in every point of the sphere. The second fundamental form of differential geometry specifies tangent plane and the normal on the surface and is invariant to parameter transformation just as the $1^{\text {st }}$ fundamental form is (Lipschutz, 1969; Struik 1988; Widder, 1989):

$$
\begin{aligned}
& I I=-d \mathbf{x} \cdot d \mathbf{N}=-d x_{i} \cdot d N_{i}=-\left(\mathbf{x}_{u} d u+\mathbf{x}_{v} d v\right) \cdot\left(\mathbf{N}_{u} d u+\mathbf{N}_{v} d v\right)= \\
& =e d u^{2}+2 f d u d v+g d v^{2} \geq 0
\end{aligned}
$$

In the case of sphere, we obtain:

$$
\begin{aligned}
& \mathbf{x}_{u u}=\mathbf{x}_{\phi \phi}=(-R \cdot \cos \phi \cdot \cos \lambda) \cdot \mathbf{i}+(-R \cdot \cos \phi \cdot \sin \lambda) \cdot \mathbf{j}+(-R \cdot \sin \phi) \cdot \mathbf{k} \\
& \mathbf{x}_{u v}=\mathbf{x}_{\phi \lambda}=(R \cdot \sin \phi \cdot \sin \lambda) \cdot \mathbf{i}+(-R \cdot \sin \phi \cdot \cos \lambda) \cdot \mathbf{j}+(0) \cdot \mathbf{k} \\
& \mathbf{x}_{v v}=\mathbf{x}_{\lambda \lambda}=(-R \cdot \cos \phi \cdot \cos \lambda) \cdot \mathbf{i}+(-R \cdot \sin \phi \cdot \sin \lambda) \cdot \mathbf{j}+(0) \cdot \mathbf{k} \\
& \text { where, } e=-\mathbf{x}_{u} \cdot \mathbf{N}_{u}=\mathbf{x}_{u u} \cdot \mathbf{N} \quad f=\mathbf{x}_{u v} \cdot \mathbf{N} \quad g=-\mathbf{x}_{v} \cdot \mathbf{N}_{v}=\mathbf{x}_{v v} \cdot \mathbf{N}
\end{aligned}
$$


Finally, we obtain:

$e=\mathbf{x}_{\phi \phi} \cdot \mathbf{N}=R \quad f=\mathbf{x}_{\phi \lambda} \cdot \mathbf{N}=0 \quad g=\mathbf{x}_{\mu \lambda} \cdot \mathbf{N}=R \cos ^{2} \phi$

The normal curvature on the surface is given as (Goetz, 1970; Lipshutz, 1969; Struik, 1988):

$\kappa_{n}=\frac{I I}{I}=\frac{-d \mathbf{x} \cdot d \mathbf{N}}{d \mathbf{x} \cdot d \mathbf{x}}=\frac{e d u^{2}+2 f d u d v+g d v^{2}}{E d u^{2}+2 F d u d v+G d v^{2}}$

In the case of sphere, we obtain:

$\kappa_{n}=\frac{e d \phi^{2}+2 f d \phi d \lambda+g d \lambda^{2}}{E d \phi^{2}+2 F d \phi d \lambda+G d \lambda^{2}}=\frac{e d \phi^{2}+g d \lambda^{2}}{E d \phi^{2}+G d \lambda^{2}}=\frac{1}{R}$

This proves that the normal curvature of sphere lies in an osculating plane and is a constant. Since the fundamental forms are proportional, every point on a sphere is umbilical or naval point (Struik, 1988). The curvature vector is:

$d \mathbf{t} / d s=\mathbf{k}=\mathbf{k}_{n}+\mathbf{k}_{g}=\left(\kappa_{n}+\kappa_{g}\right) \cdot \mathbf{N}$

It can be easily shown that geodesics are lines of shortest distance with an important property that geodesic curvature is zero (Struik, 1988). For deeper understanding of geodesics and its various applications (e.g., general theory of relativity) consult Goetz (1970), Kreyszig (1964), Lipschutz (1969), Oprea, 2007, Struik (1988), Wrede (1972), etc. The important mean and Gauss (total) curvatures are defined as (Lipschutz, 1969; Struik, 1988):

$$
\begin{aligned}
& \bar{\kappa}=\frac{\kappa_{1}+\kappa_{2}}{2}=\frac{E g-2 f F+e G}{2\left(E G-F^{2}\right)}=\frac{1}{R} \\
& \kappa_{G}=\kappa_{1} \cdot \kappa_{2}=\frac{e g-f^{2}}{E G-F^{2}}=\frac{1}{R^{2}}
\end{aligned}
$$

All the points on the sphere are thus elliptic umbilical points. Gauss curvature is an invariant property of the surface. The surface of the sphere can be found calculated from the $2^{\text {nd }}$ Fundamental theorem: 


$$
\begin{aligned}
& A=\iint_{S} \sqrt{E G-F^{2}} d \phi d \lambda=\iint_{S} R^{2} \cos \phi d \phi d \lambda= \\
& =R^{2} \int_{-\pi / 2}^{\pi / 2} \cos \phi d \phi \int_{-\pi}^{\pi} d \lambda=4 \pi R^{2}
\end{aligned}
$$

Since the spherical-average terrestrial radius is $6,371 \mathrm{~km}$, the surface area of the perfectly smooth planet Earth is about 510 million $\mathrm{km}^{2}$ or 197 million $\mathrm{SM}^{2}$ (148.7 million $\mathrm{NM}^{2}$ ). Land mass is about $30 \%$ or 150 million $\mathrm{km}^{2}$ or about 58 million $\mathrm{SM}^{2}$ (43.73 million $\mathrm{NM}^{2}$ ). Five spatially largest countries: Russia $\left(17,075,200 \mathrm{~km}^{2}\right)$, Canada $(9,984,670)$, USA $(9,826,630)$, China $(9,596,960)$, and Brazil $(8,511,965)$ cover almost 55 million $\mathrm{km}^{2}$ or more than $1 / 3$ of the entire land mass.

The volume of the sphere is obtained by integrating infinitesimal volume in spherical coordinate system:

$$
V=\iiint_{V} d V=\int_{0}^{R} \int_{-\pi-\pi / 2}^{\pi} \int_{0}^{\pi / 2} r d \phi \cdot r \cos \phi d \lambda \cdot d r=\int_{0}^{R} r^{2} d r \int_{-\pi}^{\pi} d \lambda \int_{-\pi / 2}^{\pi / 2} \cos \phi d \phi=\frac{4}{3} \pi R^{3}
$$

The volume of spherical Earth is accordingly $1.0832 \times 10^{21} \mathrm{~m}^{3}$. The mass and average density of Earth is easily calculated from the gravitational data. 


\section{Appendix $B$}

Geodesics on a sphere - Variational calculus problem

Geodesic lines or geodesics are defined as lines (curves) of shortest length on any surface (Greenwood, 1997; Lanczos, 1986; Lass, 2009; Smith, 1998; Struik, 1988). Struik (1988) also provides a more general definition of geodesics as curves of zero geodesic curvature. For a sphere this simply means that geodesics are "straight" lines with the entire curvature in the osculating plate and no curvature in the rectifying plane. This also implies absence of any torsion for Orthodrome curves on sphere (Struik, 1988). For example, straight lines are geodesic curves on planar surfaces and that can be easily mathematically proven (Smith, 1998). Quite generally, geodesic lines can be derived using the EulerLagrange (E-L) equations of calculus of variations (Fox, 1987; Greenwood, 1997; Lanczos, 1986; Lass, 2009; Smith, 1998; Weber and Arfken, 2004). On spheres, the geodesic lines are GCs (Greenwood, 1997; Lanczos, 1986; Lass, 2009; Smith, 1998; Weber and Arfken, 2004). GC distances are also called Orthodromes (Weintrit and Kopacz, 2011) GEs are approximately geodesic lines on ellipsoids of revolutions (Bowring, 1984; Sjöberg, 2012; Tseng and Lee, 2010; Williams, 1986).

Although, GCs are shortest lines (geodesics) on a perfect sphere, the tangent (heading) is constantly changing in spherical coordinate system, which historically presented a problem for maritime and long-range air navigation. As a matter of fact, Riemann's geometry can be interpreted on a sphere by taking GCs as straight lines (Struik, 1988). In Euclidian geometry the length of the parametric curve between two points is:

$$
L=R \int_{t_{1}}^{t_{2}} \sqrt{\left(\frac{d x}{d t}\right)^{2}+\left(\frac{d y}{d t}\right)^{2}+\left(\frac{d z}{d t}\right)^{2}} d t
$$

Using the first fundamental form of differential geometry for a sphere (Goetz, 1970; Kreyszig, 1964; Lipschutz, 1969; Oprea, 2007; Struik, 1988) or by direct differentiation from Equation (B1), the length of a curve along the spherical surface is a parametric curve where the longitude is a function of latitude (Equation A10):

$$
L=R \int_{\phi_{1}}^{\phi_{2}} \sqrt{1+\cos ^{2} \phi \cdot\left(\frac{d \lambda}{d \phi}\right)^{2}} d \phi \geq 0
$$


The integral given by Equation (B2) belongs to a class of (incomplete) elliptic integrals of the second kind (Abramowitz and Stegun, 1984; Byrd and Friedman, 1954; Dwight, 1961; Jahnke and Emde, 1945; Spiegel and Liu, 1999):

$$
E(k, \phi)=\int_{0}^{\phi} \sqrt{1-k^{2} \cos ^{2} \theta} d \theta
$$

Elliptic integrals originated in problems of rectification of elliptical orbital arcs. In general, they do not have analytical (closed-form) solution (Byrd and Friedman, 1954). The goal is now to find a curve (out of infinitely many possible) on a sphere with $\lambda=\lambda(\phi)$ so that length $L(\lambda)$ is minimized between the starting $P_{1}$ $\left(\phi_{1}, \lambda_{1}\right)$ and the end point $P_{2}\left(\phi_{2}, \lambda_{2}\right)$. Calculus of variations was developed to precisely deal with these kind of problems. For more details on variational and optimization methods/principles and its applications in physics and engineering a reader could consult references used here, such as, Fox (1987), Greenwood (1997), Lanczos (1986), Smith (1998), and Weber and Arfken (2004).

The variational problem of finding the shortest distance (geodesic) on a spherical surface between two known points is formally known as an inverse or $2^{\text {nd }}$ geodesic problem (Bomford, 1983; Vaníček and Krakiwsky, 1986). Thus, Equation (A4), can be formally transformed into variational problem involving functional L( $\lambda)$ (Fox, 1987: Smith, 1998):

$$
L(\lambda)=\int_{\phi_{0}}^{\phi_{1}} F\left[\phi, \lambda_{\phi}(\phi)\right] d \phi \quad F(\phi, w)=R \cdot \sqrt{1+\cos ^{2} \phi \cdot w^{2}} \quad w=\frac{d \lambda(\phi)}{d \phi}=\lambda_{\phi}
$$

Here, a particular curve $\lambda(\phi)$ resulting in shortest length $L$ defines a geodesic on a spherical surface, i.e., GC or Orthodrome with two ends anchored in known starting and ending points. To solve this problem we use the powerful Euler-Lagrange equations (Fox, 1987; Greenwood, 1997; Lanczos, 1986; Lass, 2009; Smith, 1998; Weber and Arfken, 2004):

$$
\frac{d}{d x}\left(\frac{\partial F}{\partial y^{\prime}}\right)-\frac{\partial F}{\partial y}=0 \quad F=F\left[x, y(x), y^{\prime}(x)\right] \quad y_{x}(x)=\frac{d y(x)}{d x}=y^{\prime}
$$

When E-L equations are satisfied presents sufficient and necessary condition to make the following integral (functional) stationary (Lanczos, 1986): 
$J(y(x))=\int_{a}^{b} F\left(x, y, y^{\prime}\right) d x \quad y(a)=\alpha \quad y(b)=\beta$

The problem of geodesics on a sphere reduces to the following E-L equations:

$\frac{d}{d \phi} \frac{\partial F\left\lfloor\phi, \lambda_{\phi}(\phi)\right\rfloor}{\partial \lambda_{\phi}}-\frac{\partial F\left\lfloor\phi, \lambda_{\phi}(\phi)\right\rfloor}{\partial \lambda}=0$

Since there is no direct dependence on longitude (meridian), i.e., $\partial F / \partial \lambda=0$, the variational problem reduces to simple:

$\frac{d}{d \phi} \frac{\partial F\left[\phi, \lambda_{\phi}(\phi)\right]}{\partial \lambda_{\phi}}=\frac{d}{d \phi} \frac{\partial F(\phi, w)}{\partial w}=0 \quad \Rightarrow \quad \frac{\partial F(\phi, w)}{\partial w}=$ const

Using definitions from Equation (A5), we obtain:

$\frac{\partial F(\phi, w)}{\partial w}=R \frac{w \cdot \cos ^{2} \phi}{\sqrt{1+w^{2} \cos ^{2} \phi}}=$ const.

We can now extract unknown meridional dependence on latitude:

$$
w=\frac{d \lambda(\phi)}{d \phi}=\frac{a}{\cos \phi \cdot \sqrt{\cos ^{2} \phi-a^{2}}} \quad a=\text { const } .
$$

Integrating Equation (A11) results in:

$\lambda(\phi)=\int \frac{a \cdot d \phi}{\cos \phi \cdot \sqrt{\cos ^{2} \phi-a^{2}}}+b$

The analytic solution of this integral can be obtained by using several different substitution methods (Dym and Shames, 2013; Fox, 1987; Oprea, 2007; Smith, 1998). First, the integral in Equation (B10) will be transformed into using trigonometric relationship, $\sec ^{2} \phi=1+\tan ^{2} \phi$ :

$\lambda(\phi)=\int \frac{a \cdot \sec ^{2} \phi \cdot d \phi}{\sqrt{1-a^{2} \cdot \sec ^{2} \phi}}+b=a \cdot \int \frac{\sec ^{2} \phi \cdot d \phi}{\sqrt{1-a^{2}-a^{2} \cdot \tan ^{2} \phi}}+b$ 
Introducing substitution (Oprea, 2007):

$$
w=\frac{a}{\sqrt{1-a^{2}}} \cdot \tan \phi=c \cdot \tan \phi \quad d w=\frac{a}{\sqrt{1-a^{2}}} \cdot \sec ^{2} \phi \cdot d \phi=c \cdot \sec ^{2} \phi \cdot d \phi
$$

The integral in Equation (B11) becomes:

$\lambda(\phi)=\int \frac{d w}{\sqrt{1-w^{2}}}+b$

Utilizing another substitution $w=\sin \varphi$ (Oprea, 2007) and then backsubstitution to the original variable for latitude, the integral in Equation (B12) becomes:

$$
\lambda(\phi)=\sin ^{-1}\left(\frac{a \cdot \tan \phi}{\sqrt{1-a^{2}}}\right)+b
$$

The geodesic on the circle is restricted (constrained) with the curve given parametrically (longitude as a function of latitude) as:

$$
\sin [\lambda(\phi)-b]=\frac{a}{\sqrt{1-a^{2}}} \cdot \tan \phi=c \cdot \tan \phi \quad a, b, c=\text { const }
$$

The unknown constants " $b$ " and " $c$ " can be evaluated from the known anchor points $P_{1}$ and $P_{2}$ satisfying:

$$
b=\lambda_{1}-\sin ^{-1}\left(c \cdot \tan \phi_{1}\right)=\lambda_{2}-\sin ^{-1}\left(c \cdot \tan \phi_{2}\right) \quad c=\frac{\sin \left(\lambda_{1}-b\right)}{\tan \phi_{1}}=\frac{\sin \left(\lambda_{2}-b\right)}{\tan \phi_{2}}
$$

These are two simultaneous transcendental (nonlinear) equations that can be solved numerically for unknowns: $b$ and $c$. Once the constants are known there is a unique (unless conjugate points) shortest curve (Orthodrome arc) that passes between two arbitrary points on the sphere. By expanding trigonometric functions in Equation (B14), we obtain:

$\cos \phi \cdot \sin \lambda \cdot \cos b-\cos \phi \cdot \cos \lambda \cdot \sin b=c \cdot \sin \phi$

Using spherical coordinate system definitions from Equation (A1), we obtain: 
$x \cdot \sin b-y \cdot \cos b+z \cdot c=0 \Rightarrow A \cdot x+B \cdot y+C \cdot z=0$

where, $A=\sin b \quad B=-\cos b \quad C=\frac{a}{\sqrt{1-a^{2}}}=c$.

This is the special case of the general equation of the plane (Hall, 1968, Spiegel and Liu, 1999):

$$
A \cdot x+B \cdot y+C \cdot z+D=0
$$

The plane described with Equation (B16) is passing through the center of the sphere $P_{0}(0,0,0)$ and the two points (anchors) on the sphere, $P_{1}\left(X_{1}, Y_{1}, Z_{1}\right)$ and $P_{2}\left(X_{2}, Y_{2}, Z_{2}\right)$ implying $D=0$ (Bronstein and Semendjajew, 1989; Hall, 1968; Olza et al., 1974; Spiegel and Liu, 1999). The same final result was also obtained by Dym and Shames (2013), Fox (1987), Oprea (2007), and Smith (1998). This plane which intersects with the sphere forms GC or Orthodrome. For antipodal (conjugate) points there are infinitely many GCs.

The radii for the two points $P_{1}$ and $P_{2}$ laying on the plane (Equation B16) in orthonormal Cartesian coordinate system have the direction cosines (Hall, 1968, Spiegel and Liu, 1999):

$$
\begin{array}{lll}
l=\cos \alpha=\frac{x-x_{0}}{R}=\frac{x}{R} & m=\cos \beta=\frac{y-y_{0}}{R}=\frac{y}{R} & n=\cos \gamma=\frac{z-z_{0}}{R}=\frac{z}{R} \\
l^{2}+m^{2}+n^{2}=1 \Rightarrow \cos ^{2} \alpha+\cos ^{2} \beta+\cos ^{2} \gamma=1 & R=\sqrt{x^{2}+y^{2}+z^{2}}
\end{array}
$$

The central angle (see also Figure B1) between the two radii for points $P_{I}$ and $P_{2}$ belonging simultaneously to the intersecting plane and the surface of the sphere is:

$\cos \sigma=l_{1} l_{2}+m_{1} m_{2}+n_{1} n_{2}=\frac{x_{1} x_{2}+y_{1} y_{2}+z_{1} z_{2}}{R^{2}} \quad 0 \leq \sigma \leq \pi$

We have thus demonstrated that an Orthodrome is a section of a GC arc, which lies in the osculating plane intersecting the center of the sphere and is a geodesic line on a perfect sphere. Such intersecting plane can always be rotated so as to coincide with the equatorial $\mathrm{GC}(z=0)$ or a meridional $\mathrm{GC}$ for which $x=0$ or $y=0$ and for which the arc-length stays invariant. 


\section{Appendix $C$}

Great Circle navigation on a perfect sphere

Let us use a spherical coordinate system with a traditional notions of latitude $\phi$ and longitude $\lambda$. For each angle of latitude there is also a corresponding angle of complementary latitude or co-latitude $\delta$ (often called polar distance). For a perfect homogeneous sphere the geocenter, geodetic (geographic) center, and barycenter are all in the same point. A schematic of a vector point on a smooth spherical surface in spherical coordinates is shown in Figure C1. The plane intersecting the sphere through the center and an arbitrary GC are shown as well. Transformation between the Cartesian coordinate system $(\mathrm{x}, \mathrm{y}, \mathrm{z})$ with the orthonormal vector basis $(\mathbf{i}, \mathbf{j}, \mathbf{k})$ and the spherical coordinates on a unit sphere is:

$$
\begin{array}{lll}
x=\cos \phi \cdot \cos \lambda & y=\cos \phi \cdot \sin \lambda & z=\sin \phi \\
-\pi / 2 \leq \phi \leq \pi / 2 & -\pi \leq \lambda \leq \pi &
\end{array}
$$
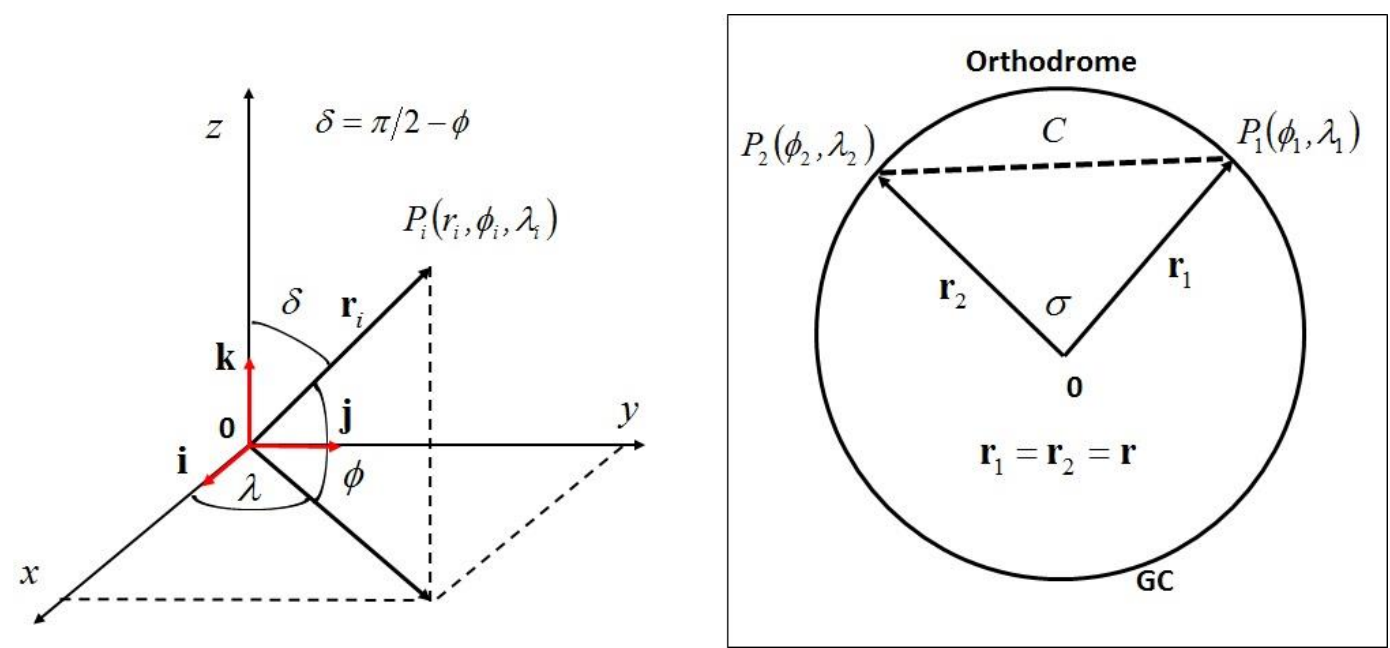

Figure $C 1$. Vector representation in spherical coordinate system and the GC lying in an osculating plane intersecting the perfect sphere through its center.

The unit vectors in the Cartesian coordinate system are orthonormal, independent, and form the basis in the Euclidian space:

$\mathbf{i} \cdot \mathbf{i}=\mathbf{j} \cdot \mathbf{j}=\mathbf{k} \cdot \mathbf{k}=1 \quad \mathbf{i} \cdot \mathbf{j}=\mathbf{i} \cdot \mathbf{k}=\mathbf{j} \cdot \mathbf{k}=0$

A radius vector of an arbitrary point on sphere of radius magnitude $R$ is: 


$$
\mathbf{r}_{i}=R \cdot\left|\begin{array}{c}
\cos \phi \cdot \cos \lambda \\
\cos \phi \cdot \sin \lambda \\
\sin \phi
\end{array}\right| \quad\left|\mathbf{r}_{i}\right|=R
$$

Two arbitrary points on a surface of a sphere with the latitude-longitude coordinates of constant radius are $P_{1}\left(R, \phi_{1}, \lambda_{1}\right)$ and $P_{2}\left(R, \phi_{2}, \lambda_{2}\right)$. Two radiusvectors originating in a geometric center now define a plane and a central angle $\sigma$. A dot (inner or scalar) product of two vectors with known norm is:

$\mathbf{r}_{1} \cdot \mathbf{r}_{2}=\left|\mathbf{r}_{1}\right| \cdot\left|\mathbf{r}_{2}\right| \cdot \cos \sigma=R^{2} \cdot \cos \sigma$

Using Equation (C2) we obtain:

$$
\begin{aligned}
& \mathbf{r}_{1} \cdot \mathbf{r}_{2}=R^{2} \cdot \cos \sigma=x_{1} \cdot x_{2}+y_{1} \cdot y_{2}+z_{1} \cdot z_{2}= \\
& =R^{2} \cdot\left[\cos \phi_{1} \cos \phi_{2}\left(\cos \lambda_{1} \cos \lambda_{2}+\sin \lambda_{1} \sin \lambda_{2}\right)+\sin \phi_{1} \sin \phi_{2}\right]
\end{aligned}
$$

This is the same result obtained previously (Equation B16). Using trigonometric addition formulas one obtains:

$$
\cos (\beta-\gamma)=\cos \beta \cos \gamma+\sin \beta \sin \gamma
$$

Substituting Equation (C5) into Equation (C4) results in:

$$
\cos \sigma=\cos \phi_{1} \cdot \cos \phi_{2} \cdot \cos \left(\lambda_{1}-\lambda_{2}\right)+\sin \phi_{1} \cdot \sin \phi_{2}
$$

The central angle can be directly derived using the Law of Cosines of the spherical trigonometry (Bowditch, 2002; Bronstein and Semendjajew, 1989; Nielsen and Vanlonkhuyzen, 1954; Olza et al., 1974; Spiegel and Liu, 1999; Todhunter, 1886):

$$
\cos \sigma=\cos \delta_{1} \cdot \cos \delta_{2}+\sin \delta_{1} \cdot \sin \delta_{2} \cdot \cos \left(\lambda_{1}-\lambda_{2}\right) \quad \delta=\frac{\pi}{2}-\phi
$$

Using familiar trigonometric conversions, the identical relationship as the one given by Equation (C6) is obtained. Taking the inverse of the cosine function (Equation $\mathrm{C6}$ or $\mathrm{C} 7$ ), delivers:

$$
\sigma=\cos ^{-1}\left[\sin \phi_{1} \cdot \sin \phi_{2}+\cos \phi_{1} \cdot \cos \phi_{2} \cdot \cos (\Delta \lambda)\right] \quad \Delta \lambda=\lambda_{1}-\lambda_{2} \quad \sigma \leq \pi
$$


A third method to estimate the central angle is based on using the chord of the GC instead the arc itself. This method is computationally the most ineffective, and is introduced solely for academic completeness. The chord is a line segment in three-dimensional Euclidian space. Using spherical coordinates on a unit sphere given by Equation (C1), the chord vector components are:

$$
\begin{aligned}
& \Delta X=\cos \phi_{2} \cdot \cos \lambda_{2}-\cos \phi_{1} \cdot \cos \lambda_{1} \\
& \Delta Y=\cos \phi_{2} \cdot \sin \lambda_{2}-\cos \phi_{1} \cdot \sin \lambda_{1} \\
& \Delta Z=\sin \phi_{2}-\sin \phi_{1}
\end{aligned}
$$

The norm (magnitude) of the chord and the central angle is:

$$
C=\sqrt{\mathbf{C} \cdot \mathbf{C}}=\sqrt{(\Delta X)^{2}+(\Delta Y)^{2}+(\Delta Z)^{2}} \quad \sigma=2 \cdot \sin ^{-1}\left(\frac{C}{2}\right)<\pi
$$

The radius vector for any point on the sphere is constant and the chord between two points form's an isosceles triangle which is split in two symmetric right triangles (see Figure $\mathrm{C} 1$ ). The GC-arc length for the angle less than or equal $180^{\circ}$ or $\pi$ between two points $P_{1}$ and $P_{2}$ on the same plane on a surface of a sphere of constant radius $R$ is thus:

$$
L_{1-2}=R \cdot \sigma=R \cdot \cos ^{-1}\left[\sin \phi_{1} \cdot \sin \phi_{2}+\cos \phi_{1} \cdot \cos \phi_{2} \cdot \cos (\Delta \lambda)\right]
$$

In practical calculations, we use northerly $(\mathrm{N})$ latitudes as positive and southerly (S) latitudes as negative. The westerly (W) longitudes are taken negative, while easterly (E) are taken positive. It will be also necessary to convert latitudes and longitudes into real numbers (angular degrees) and then possibly also convert into radians if software implementations requires it.

However, as pointed out by Sinnott (1984), the Orthodrome distance calculations for two points in proximity (e.g., closely spaced astronomical bodies on celestial sphere) using Equation (C8) may produce large errors due to the finite number of significant digits in discrete computations. Thus Sinnott (1984) suggests using the haversine formula, which was well known and used in the old navigational and astronomical tabular computations (Bowditch, 2002). The somewhat antiquated haversine and versine trigonometric functions have also been used recently in generalized wind-triangle computations and its effects on aircraft in cruise by Daidzic (2016a). We define haversine of an angle as (Daidzic, 2016; Dwight, 1961; Nielsen and Vanlonkhuyzen, 1954; Sinnott, 1984; Todhunter, 1886): 
$\operatorname{havers}(\theta)=\frac{\operatorname{vers}(\theta)}{2}=\frac{1-\cos (\theta)}{2}=\sin ^{2}\left(\frac{\theta}{2}\right) \quad \cos (\theta)+\operatorname{vers}(\theta)=1$

Using the definition given in Equation (C9) it can be easily shown that (Nielsen and Vanlonkhuyzen, 1954):

$$
\text { havers }(\sigma)=\text { havers }\left(\phi_{1}-\phi_{2}\right)+\cos \phi_{1} \cdot \cos \phi_{2} \cdot \operatorname{havers}\left(\lambda_{1}-\lambda_{2}\right)
$$

Again using Equations (C9) and (C10), the haversine (inverse haversine) formula for GC distance $\left(\Delta \phi=\phi_{1}-\phi_{2}, \Delta \lambda=\lambda_{1}-\lambda_{2}\right)$ expressed over inverse sine becomes:

$$
\begin{aligned}
& L_{1-2}=R \cdot \sigma=2 \cdot R \cdot \sin ^{-1}\left[\sqrt{\text { havers }(\Delta \phi)+\cos \phi_{1} \cdot \cos \phi_{2} \cdot \text { havers }(\Delta \lambda)}\right]= \\
& =2 \cdot R \cdot \sin ^{-1}\left[\sqrt{\sin ^{2}\left(\frac{\Delta \phi}{2}\right)+\cos \phi_{1} \cdot \cos \phi_{2} \cdot \sin ^{2}\left(\frac{\Delta \lambda}{2}\right)}\right]
\end{aligned}
$$

Haversine expression is much more accurate for very short distances unless 64-bit floating-point arithmetic computations are used implementing Equations (C7) and (C8). However, the haversine formula fails when calculating Orthodromic distances for antipodal (conjugate) points on the sphere. The angle ambiguity is avoided by using the inverse tangent function (ATAN2) found in many software implementations (Basic, Fortran, Matlab, Excel) as the arctan (arcus-tangent) function is checked in all four quadrants. Using common trigonometric relationships we can convert inverse sine (arcus sinus) into inverse tangent function and write for Orthodrome's arc central angle:

$$
\sigma=\tan ^{-1}\left\{\frac{\sqrt{\text { havers }(\Delta \phi)+\cos \phi_{1} \cdot \cos \phi_{2} \cdot \text { havers }(\Delta \lambda)}}{\sqrt{1-\left[\text { havers }(\Delta \phi)+\cos \phi_{1} \cdot \cos \phi_{2} \cdot \text { havers }(\Delta \lambda)\right]}}\right\}
$$

Equations (C11), (C14), and (C15) are working equations for Orthodrome calculations between two non-conjugate points on the spherical Earth. For very short distances, haversine Equation $(\mathrm{C} 13)$ is recommended, while for almost antipodal, inverse-cosine Equation (C11) should be used. Very short flights are mostly following constant-heading rhumb-lines and the possible error caused by implementing Equation (C11) is almost never encountered in air navigation. An average error of about $0.5 \%$ exists when using Orthodrome versus geodesics and GEs on terrestrial reference ellipsoid. The exact error varies and can be somewhat smaller or larger depending on the exact route. For a 10,000 NM non-stop flight 
one can expect an error typically of about 30-50 NM (corresponding to 4-6 minutes in high-altitude high-subsonic cruise flight), which is acceptable for most applications considering all other uncertainties. To reduce distance errors to $0.05 \%$, or less, GE on oblate ellipsoidal Earth approximation is recommended (Bowring, 1984; Sjöberg, 2012; Tseng and Lee, 2010). Accurate geodesic distances from few centimeters to $20,000 \mathrm{~km}$ on WGS-84 reference ellipsoids can be calculated using the well-known iterative algorithm of Vincenty (1975) and Karney (2013), but for air navigation purposes this is hardly needed.

To calculate all (or arbitrary many) waypoints on the GC, we use the normal vector on the osculating plane in which GC lies. Using the definition of the vector (or cross) product, we obtain the vector normal as (Ayres as Mendelson, 2009; Bronstein and Semendjajew, 1989; Hall, 1968; Olza et al., 1974; Wolper, 2001):

$$
\begin{aligned}
\mathbf{N}= & \mathbf{r}_{1} \times \mathbf{r}_{2}=\left|\begin{array}{ccc}
\mathbf{i} & \mathbf{j} & \mathbf{k} \\
x_{1} & y_{1} & z_{1} \\
x_{2} & y_{2} & z_{2}
\end{array}\right|=N_{x} \cdot \mathbf{i}+N_{y} \cdot \mathbf{j}+N_{z} \cdot \mathbf{k}= \\
& =\mathbf{i} \cdot\left(y_{1} z_{2}-y_{2} z_{1}\right)+\mathbf{j} \cdot\left(x_{2} z_{1}-x_{1} z_{2}\right)+\mathbf{k} \cdot\left(x_{1} y_{2}-x_{2} y_{1}\right)
\end{aligned}
$$

where,

$$
\mathbf{i} \times \mathbf{i}=\mathbf{j} \times \mathbf{j}=\mathbf{k} \times \mathbf{k}=0 \quad \mathbf{i} \times \mathbf{j}=\mathbf{k} \quad \mathbf{j} \times \mathbf{k}=\mathbf{i} \quad \mathbf{k} \times \mathbf{i}=\mathbf{j}
$$

After complicating vector algebra using Equation (C2), we obtain for normal vector components:

$$
\begin{aligned}
& N_{x}=R^{2} \cdot\left(\cos \phi_{1} \sin \phi_{2} \sin \lambda_{1}-\cos \phi_{2} \sin \phi_{1} \sin \lambda_{2}\right) \\
& N_{y}=R^{2} \cdot\left(\cos \phi_{2} \sin \phi_{1} \cos \lambda_{2}-\cos \phi_{1} \sin \phi_{2} \cos \lambda_{1}\right) \\
& N_{z}=-R^{2} \cdot \cos \phi_{1} \cos \phi_{2} \sin \left(\lambda_{1}-\lambda_{2}\right)
\end{aligned}
$$

Since vector normal is perpendicular to all points lying on the plane, including the points that are common to GC, then for an arbitrary vector point on an Orthodrome, we write the condition for perpendicularity of vectors:

$$
\mathbf{N} \cdot \mathbf{r}=N_{x} \cdot x+N_{y} \cdot y+N_{z} \cdot z=0
$$

By substituting the spherical coordinates from Equation (C2), we obtain: 
$\tan \phi=-\frac{N_{x} \cos \lambda+N_{y} \sin \lambda}{N_{z}} \Rightarrow \phi=\tan ^{-1}\left[-\frac{N_{x} \cos \lambda+N_{y} \sin \lambda}{N_{z}}\right]$

Thus any point on a GC segment must satisfy Equations (B15) and (C19). After substituting Equation (C17) into Equation (C19), we obtain latitude on a GC for an arbitrary longitude (taken between the starting and ending longitudes):

$\phi=\tan ^{-1}\left[\frac{\tan \phi_{1} \sin \left(\lambda-\lambda_{2}\right)+\tan \phi_{2} \sin \left(\lambda_{1}-\lambda\right)}{\sin \left(\lambda_{1}-\lambda_{2}\right)}\right] \quad \lambda_{1} \neq \lambda_{2}$

The heading (TC) is constantly changing on GC route (unless on meridians or equator). This is due to convergence of meridians $(=\Delta \lambda \cdot \sin \phi)$ toward the poles which can be observed in Figure C2. Small circle radii decrease with the cosine of latitude to become zero at the poles (see Equation A10 and its corollaries). Also shown in Figure $\mathrm{C} 2$ is Loxodrome, which is curving toward nearest pole. As a matter of fact, the Loxodrome would be spiraling infinitely around the pole, but has a finite length.

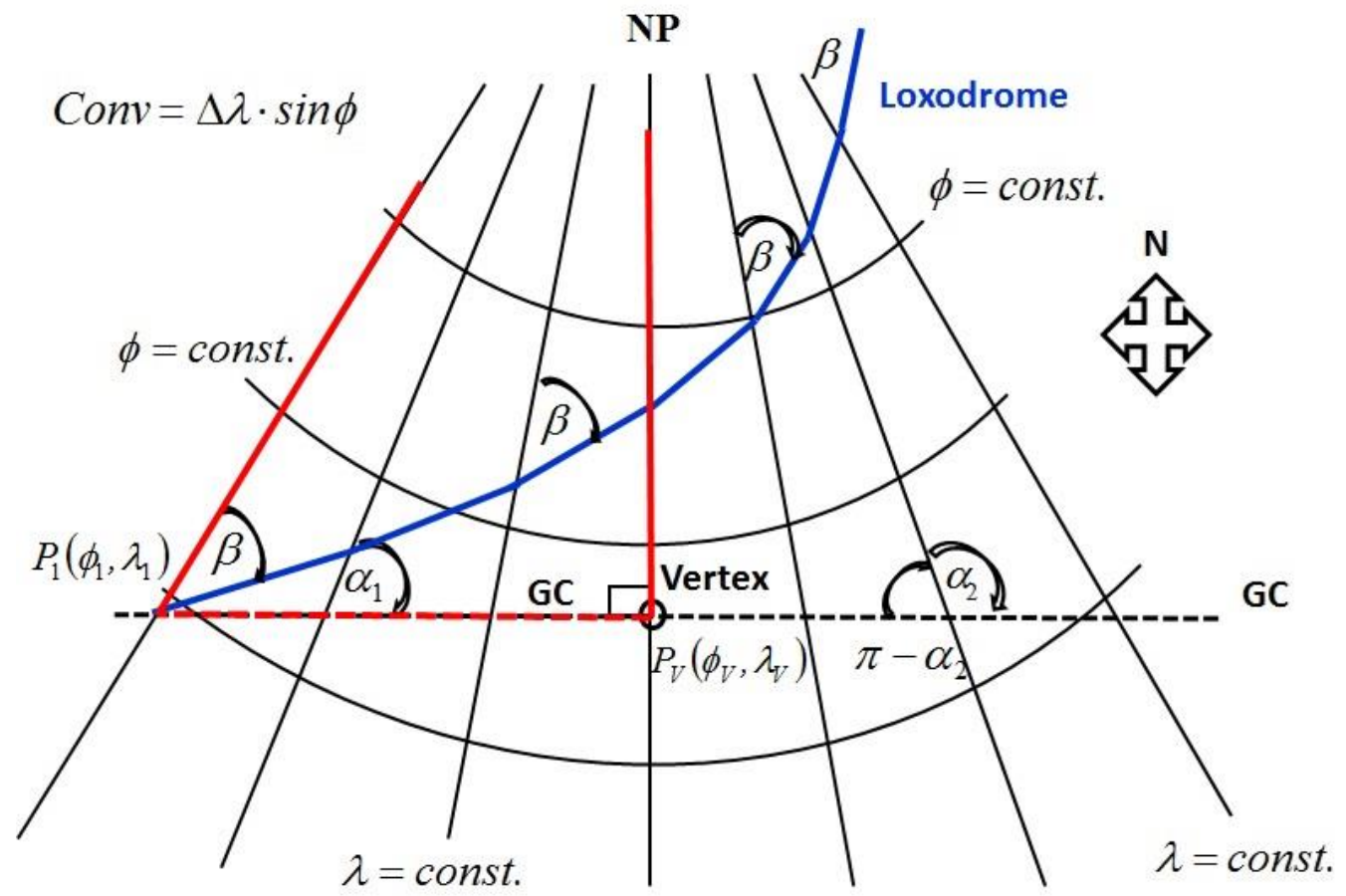

Figure $C 2$. Gnomonic projection depicting Orthodrome and Loxodrome. Not to scale. 
Using the Law of Sines in spherical trigonometry (Bronstein and Semendjajew, 1989; Danby, 1962; Nielsen and Vanlonkhuyzen, 1954; Olza et al., 1974; Spiegel and Liu, 1999; Todhunter, 1886), we can write:

$\frac{\sin \sigma}{\sin (\Delta \lambda)}=\frac{\sin \delta_{2}}{\sin \alpha_{1}}=\frac{\sin \delta_{1}}{\sin \left(\pi-\alpha_{2}\right)}=\frac{\sin \delta_{1}}{\sin \beta_{2}}$

The starting and ending (final) true courses (heading or bearing) are:

$\alpha_{1}=\sin ^{-1}\left[\frac{\sin (\Delta \lambda)}{\sin \sigma} \cos \phi_{2}\right] \quad \alpha_{2}=\sin ^{-1}\left[\frac{\sin (\Delta \lambda)}{\sin \sigma} \cos \phi_{1}\right]$

Unfortunately, this simple relationship does not resolve angle ambiguity of inverse-sine and is quite dangerous to implement trivially. But the TC information can be also obtained using the Cosine Law of spherical trigonometry (Danby, 1962; Nielsen and Vanlonkhuyzen, 1954; Todhunter, 1886), resulting in:

$$
\begin{aligned}
& \cos \alpha_{1}=\frac{\cos \delta_{2}-\cos \sigma \cdot \cos \delta_{1}}{\sin \sigma \cdot \sin \delta_{1}}=\frac{\sin \phi_{2}-\sin \phi_{1} \cdot \cos \sigma}{\cos \phi_{1} \cdot \sin \sigma} \\
& \cos \beta_{2}=-\cos \alpha_{2}=\frac{\sin \phi_{1}-\sin \phi_{2} \cdot \cos \sigma}{\cos \phi_{2} \cdot \sin \sigma}
\end{aligned}
$$

For numerical accuracy and resolving the angle ambiguity using periodic trigonometric functions it is always better to use inverse-tangent function which in software implementation is typically coded as ATAN2(y,x) or similar (in True Basic an intrinsic function ANGLE (x,y) is used). Quadrants are shown in Figure C3. In terms of inverse tangent function by combining Equations (C22) and (C23) and doing basic trigonometric transformations, one obtains (see Figures $\mathrm{C} 2$ and C3):

$$
\begin{aligned}
& \alpha_{1}=\tan ^{-1}\left[\frac{\sin (\Delta \lambda)}{\cos \phi_{1} \cdot \tan \phi_{2}-\sin \phi_{1} \cdot \cos (\Delta \lambda)}\right] \\
& \alpha_{2}=\tan ^{-1}\left[\frac{\sin (\Delta \lambda)}{-\cos \phi_{2} \cdot \tan \phi_{1}+\sin \phi_{2} \cdot \cos (\Delta \lambda)}\right] \quad \beta_{2}=\pi-\alpha_{2}
\end{aligned}
$$

Utilizing the Law of Sines, we can also find the very important point along GC route called the vertex (Jeppesen, 2007; Underdown and Palmer, 2001; Wolper, 2001). Vertex is the point of highest latitude ( $\mathrm{N}$ or $\mathrm{S}$ ) for which the 
instantaneous $\mathrm{TC}$ is equal to $90^{\circ}$ or $270^{\circ}$ and is illustrated in Figure C3 together with the GC segments. Using the Law of Sines as given in Equation (C21), we can write from the second and third equality:

$\frac{\sin \delta_{2}}{\sin \alpha_{1}}=\frac{\sin \delta_{1}}{\sin \left(\pi-\alpha_{2}\right)} \Rightarrow \sin \alpha_{1} \cos \phi_{1}=\sin \alpha_{2} \cos \phi_{2}$

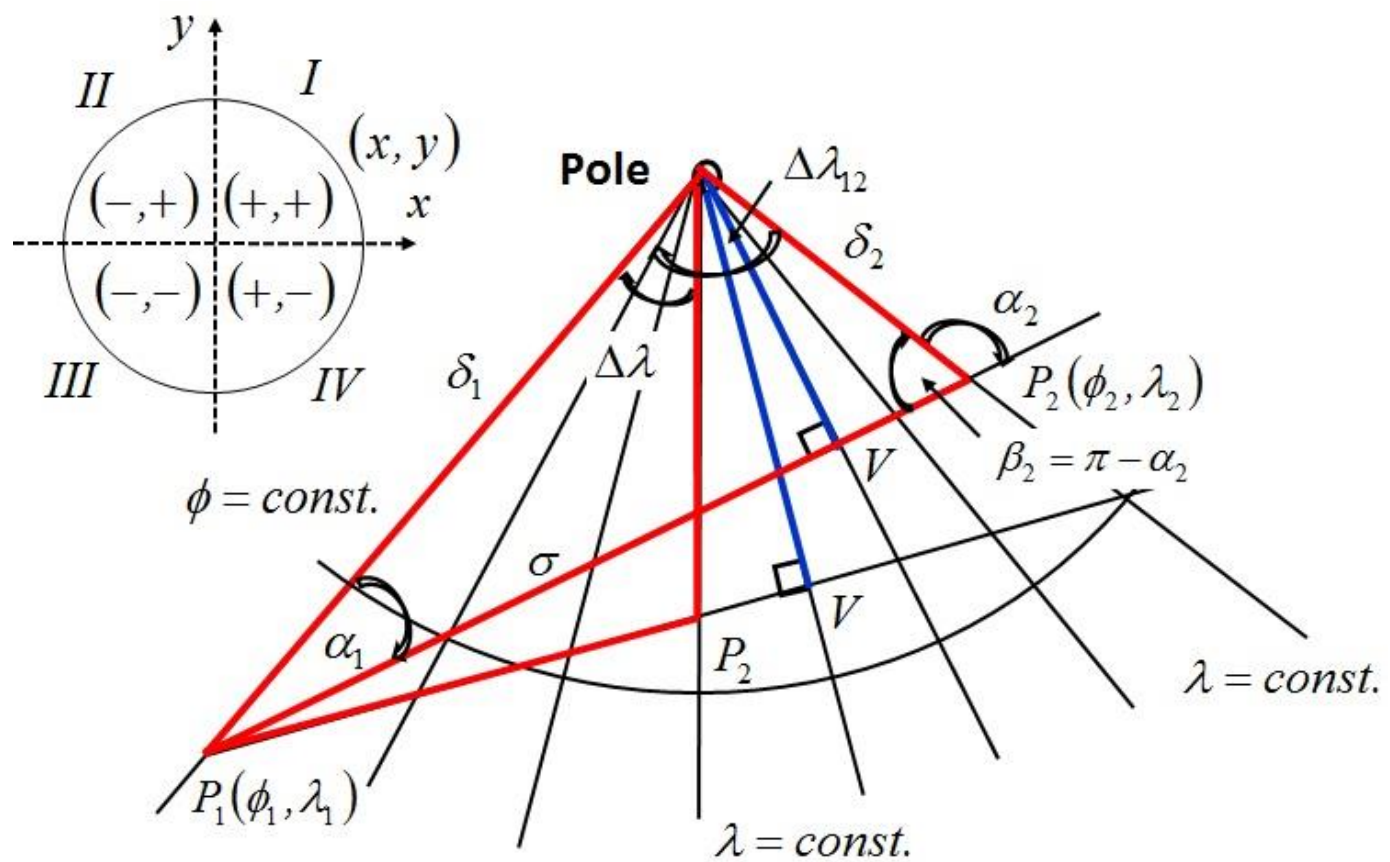

Figure C3. The location of vertex on GC-arc routes using the polar gnomonic projection. Not to scale.

This expression is sometimes called Clairaut's relation or theorem (Struik, 1988), but for spheres it actually comes originally from many Arabic and Iranian/Persian scholars in the period from $9^{\text {th }}$ to $13^{\text {th }}$ century, such as, Al-Jayyani and Nasir al-din al-Tusi (Struik, 1987) who, by-the-way, have fully developed planar and spherical trigonometry. Equation (C25) is valid for any two points on a GC. It will also be valid for the point where the GC crosses equator (going $\mathrm{N}$ or $\mathrm{S})$. Since the latitude at the equator is $0^{\circ}$, and the TC at vertex is $\sin (\pi / 2)=1$ or $\sin (3 \pi / 2)=-1$, we may write:

$\sin \alpha_{e q}=\sin \alpha_{1} \cos \phi_{1}=\sin \alpha_{2} \cos \phi_{2}=\cos \phi_{\text {vertex }}$ 
The latitude ( $\mathrm{N}$ or $\mathrm{S}$ ) of the vertex is thus:

$$
\phi_{\text {vertex }}=\cos ^{-1}\left|\sin \alpha_{e q}\right|=\cos ^{-1}\left|\sin \alpha_{1} \cdot \cos \phi_{1}\right|=\cos ^{-1}|\sin \alpha \cdot \cos \phi|
$$

A GC which is not an equator or meridian (with anti-meridian) will have two vertices ("higher" and "lower" or N and S). The longitude of the vertex is shifted by $90^{\circ}$ from the longitude at which GC crosses Equator. The longitude of the vertex can be easily derived and yields:

$$
\lambda_{\text {vertex }}=\lambda_{1} \pm \cos ^{-1}\left(\frac{\tan \phi_{1}}{\tan \phi_{\text {vertex }}}\right)
$$

We can easily prove the relationship between the vertex and Equator's $(E q)$ crossing longitudes by using:

$\tan \phi_{E q}=\cos \left(\Delta \lambda_{E q-v e r t e x}\right) \cdot \tan \phi_{v e r t e x}$

All vertex geometric properties can be derived using Napier's rules of right-angled spherical triangle (Bowditch, 2002; Nielsen and Vanlonkhuyzen, 1954; Olza et al., 1974; Todhunter, 1886). Since Equator is excluded as a GC route and has undefined vertex it must be $\tan \phi_{\text {vertex }} \neq 0$. On the other hand, we have $\tan \phi_{E q}=0$. Hence the solution is:

$$
\cos \left(\Delta \lambda_{E q-\text { vertex }}\right)=0 \quad \Delta \lambda_{E q-\text { vertex }}= \pm(2 n+1) \frac{\pi}{2} \quad n=0,1,2, \ldots
$$

Finally, we have defined and derived all working equations necessary for $\mathrm{GC}$ route computations on spherical Earth. In general, if the Orthodrome route is along the equator or any meridian (which are GCs on spherical Earth) then the problem is trivial and many of the here derived equations will fail. For example, vertex is undefined for the $\mathrm{GC}$ along the Equator or any meridian. However, the TCs are then simply equal to $0^{\circ} / 180^{\circ}$ (along any meridian) or $90^{\circ} / 270^{\circ}$ (along Equator) and the Orthodrome is equal to change in longitude multiplied by the spherical Earth radius $(6,371 \mathrm{~km})$ when along equator. Similar reasoning will apply for any meridian and its anti-meridian. 


\section{Appendix D}

\section{Short distance Great Circle navigation on a perfect sphere}

As mentioned previously the cosine-formula for GC (Equation C8) fails for very short arcs. While mathematically exact, the problem occurs with the finite number of digits representation as was illustrated by Sinnott (1984). Essentially for angular distances of less than 1 arc-minute it is better to use haversine formula (Equations $\mathrm{C} 14$ and C15) although modern 64-bit floating point arithmetic's can push the envelope to less than 1 arc-second. Such small angular distances have important astronomical, but practically no air navigation applications.

For short distances on Earth (around $20 \mathrm{~km}$ or less), it is acceptable to neglect Earth's spheroidal shape and utilize planar-trigonometry. Here, we will distinguish two special cases (1) Polar-coordinates flat-Earth formula used with polar azimuthal projections for high latitudes, and (2) planar projections using simple Pythagoras theorem (Euclidian space). Close to the geographic poles (NP or SP), the polar projections (De Remer and McLean, 1998; Jeppesen, 2007; Struik, 1988; Underdown and Palmer, 2001) will result in meridians being represented as radials coming out of poles, while the lines of latitude are concentric circles with separation distance between them changing depending on the type of projection. The polar stereographic azimuthal projections is illustrated in Figure D1. Polar gnomonic would be very similar, but the distance between the lines of latitude will be increasing rapidly (Equator is in infinity). Orthographic projection has center of projection in infinity (Figure D2). The lines of latitude are decreasingly spaced concentric circles. Conjugate hemisphere is not represented.
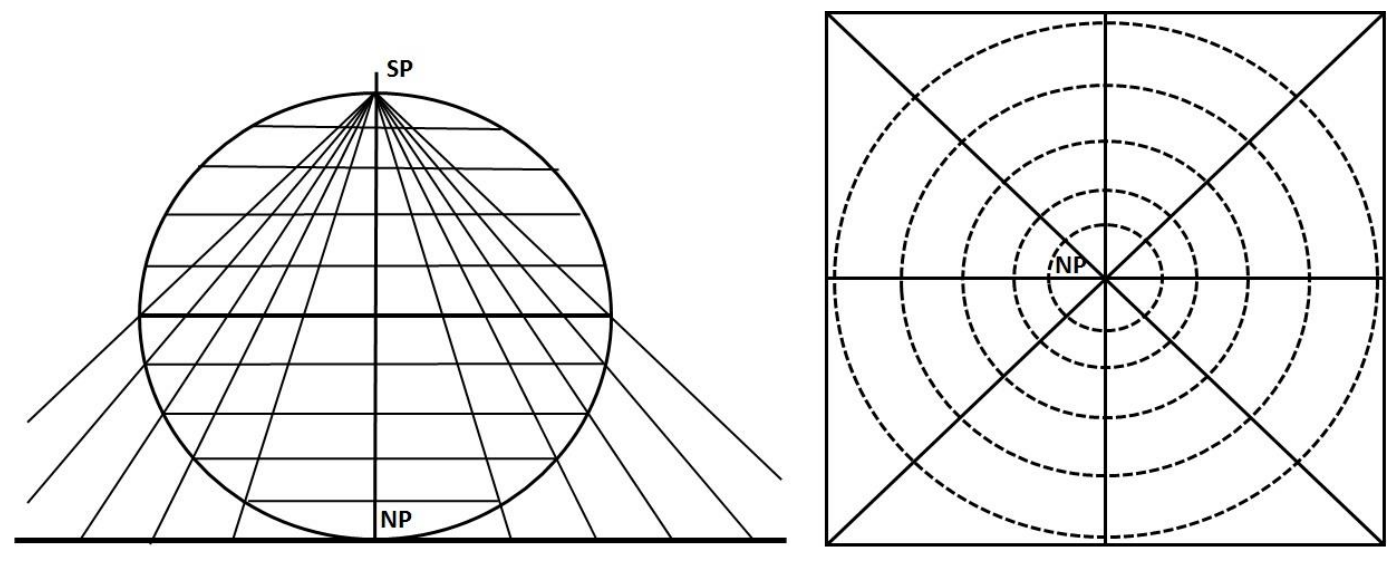

Figure D1. Polar stereographic azimuthal projection of Earth. Not to scale. 

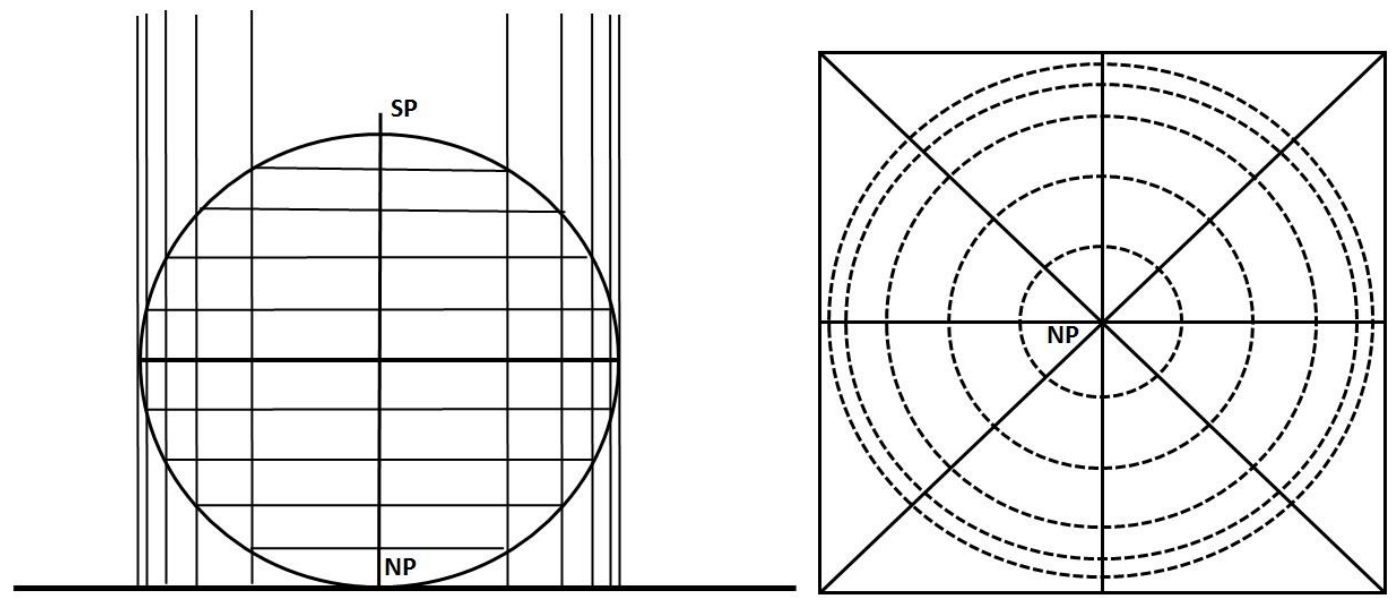

Figure D2. Polar orthographic azimuthal (plane) projection of Earth. Not to scale.

Polar-coordinates azimuthal projection formula originates from the Law of Cosines of planar trigonometry (Olza et al, 1974; Spiegel and Liu, 1999) as illustrated in Figure D3:

$$
\sigma_{1-2}=\sqrt{\delta_{1}^{2}+\delta_{2}^{2}-2 \cdot \delta_{1} \cdot \delta_{2} \cdot \cos \left(\Delta \lambda_{1-2}\right)} \quad \phi+\delta=\frac{\pi}{2}
$$
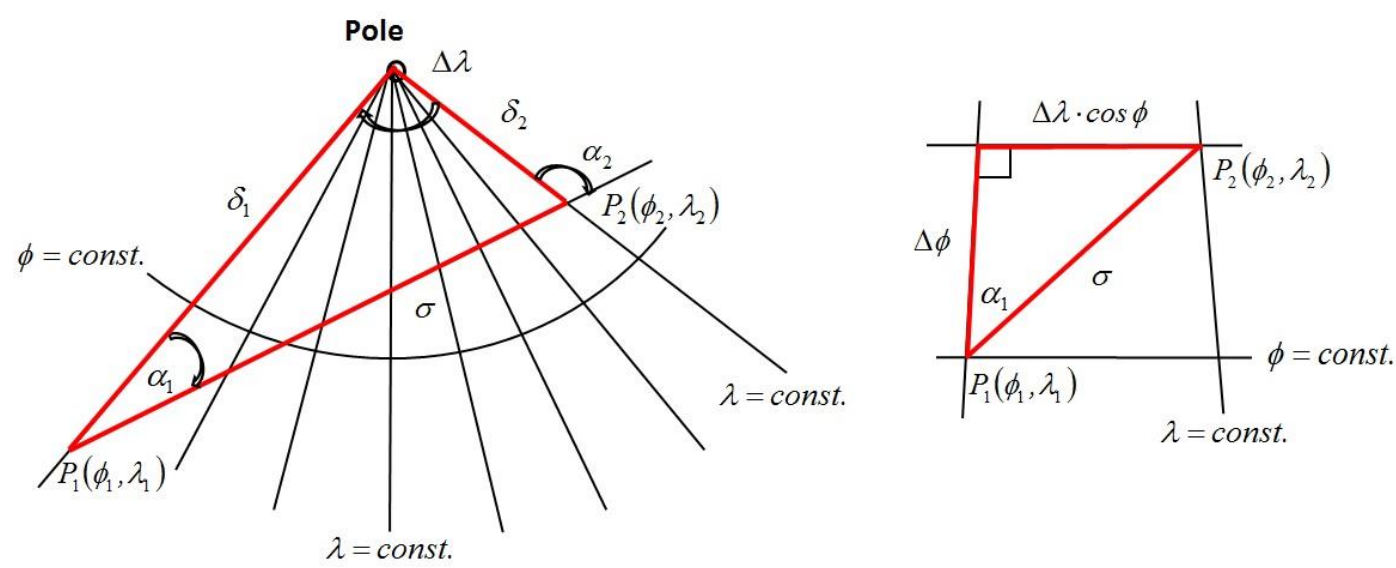

Figure D3. Polar azimuthal gnomonic projection with polar coordinates and rectangular azimuthal projection for short-distance computations.

Substituting co-latitudes (polar distances) with geocentric latitudes, we obtain for the central angle and the chord distance approximating GC arc: 


$$
\begin{aligned}
\sigma_{1-2} & =\sqrt{\left(\frac{\pi}{2}-\phi_{1}\right)^{2}+\left(\frac{\pi}{2}-\phi_{2}\right)^{2}-2 \cdot\left(\frac{\pi}{2}-\phi_{1}\right) \cdot\left(\frac{\pi}{2}-\phi_{2}\right) \cdot \cos \left(\lambda_{1}-\lambda_{2}\right)} \\
L_{1-2} & =\bar{R}_{E} \cdot \sigma_{1-2} \quad \bar{R}_{E}=6,371,000 \mathrm{~m}
\end{aligned}
$$

The closer the straight-line course in polar azimuthal projection is to the poles the more accurate the approximation is. We can also derive TCs at points 1 (departure) and 2 (destination) using the same law-of-cosines:

$$
\alpha_{1}=\cos ^{-1}\left\{\frac{\sigma_{1-2}^{2}+\left(\pi / 2-\phi_{1}\right)^{2}-\left(\pi / 2-\phi_{2}\right)^{2}}{2 \cdot\left(\pi / 2-\phi_{1}\right)^{2} \cdot \sigma_{1-2}}\right\} \quad \alpha_{2}=\alpha_{1}+\Delta \lambda
$$

A simple right-angle approximation (Pythagoras theorem) can be used for lower latitudes and very short distances:

$$
\sigma_{1-2}=\sqrt{\left(\phi_{1}-\phi_{2}\right)^{2}+\left(\lambda_{1}-\lambda_{2}\right)^{2}} \quad L_{1-2}=\bar{R}_{E} \cdot \sigma_{1-2} \quad \alpha_{1}=\tan ^{-1}\left(\frac{\lambda_{1}-\lambda_{2}}{\phi_{1}-\phi_{2}}\right)
$$

Essentially the convergence of the meridians and the curvature of the parallels is neglected. A comprehensive analysis of the deviation from the various GC formulas was not conducted as this presents a significant effort in itself. A somewhat improved oblique stereographic azimuthal projection short-distance GC that partially takes into account convergence and latitude is:

$$
\sigma_{1-2}=\sqrt{\left(\phi_{1}-\phi_{2}\right)^{2}+\cos ^{2}\left(\frac{\phi_{1}+\phi_{2}}{2}\right) \cdot\left(\lambda_{1}-\lambda_{2}\right)^{2}} \quad L_{1-2}=\bar{R}_{E} \cdot \sigma_{1-2}
$$




\section{Appendix E}

Rhumb line navigation and the Loxodrome

Maintaining constant angle (headings, courses, bearings) in relationship to terrestrial meridians is called rhumb-line navigation. Rhumbs are part of curves called Loxodromes. Rhumb line navigation does not deliver shortest distances on spherical or spheroidal Earth. A Loxodrome is a very interesting curve that for any heading other than North-South and East-West (coincides with Orthodromes) ends up spiraling around the NP and SP (Alexander, 2004; Lipshutz, 1969; Struik, 1988). Loxodrome is a straight line on a Mercator cylindrical conformal projection. For example, on a polar stereographic conformal projection a Loxodrome becomes a logarithmic spiral (Lipshutz, 1969; Struik, 1988; Alexander, 2004) about respective poles. Conformal Mercator cylindrical chart provides for uniform stretching throughout the chart (N-S and E-W) resulting in preservation of local angles (conformality) delivering isotropic Representative Fraction (RF) or scale. The local and the total stretching from the Equator on spherical Earth is expressed mathematically as:

$$
\begin{aligned}
& \psi(\phi)=\sec \phi=\frac{1}{\cos \phi} \\
& \bar{\psi}(\phi)=\int_{0}^{\phi} \sec \phi d \phi=\ln (\sec \phi+\tan \phi)=\ln \left[\tan \left(\frac{\phi}{2}+\frac{\pi}{4}\right)\right]
\end{aligned}
$$

The method of calculating total stretching in the medieval time was defacto numerical integration and was referred to by Edward Wright as "perpetual addition of Secants" (Alexander, 2004). Naturally for Mercator cylindrical projections with the line of contact coinciding with the Equator, the poles (singular points on sphere) cannot be represented as the total stretching goes to infinity. One could use oblique or transverse cylindrical projections instead to depict poles (De Remer and McLean, 1998; Jeppesen, 2007; Underdown and Palmer, 2001).

Using Equation (A11) with one vector being collinear with the lines of longitude $(\lambda=$ const., $\delta \lambda=0)$, while the other represents Loxodrome on a sphere, one obtains:

$$
\cos \alpha=E \frac{d \phi}{d s} \frac{\delta \phi}{\delta s}=R \frac{d \phi}{d s} \quad \delta s=\sqrt{E \cdot \delta \phi^{2}}=R \cdot \delta \phi
$$


Using the first fundamental form for sphere this can be written as:

$$
\cos ^{2} \alpha \cdot d s^{2}=R^{2} \cdot d \phi^{2} \Rightarrow \frac{d \phi}{\cos \phi}= \pm \operatorname{ctg} \alpha \cdot d \lambda
$$

Integrating Equation (E3), we obtain:

$$
\int_{\phi_{1}}^{\phi_{2}} \frac{d \phi}{\cos \phi}= \pm \operatorname{ctg} \alpha \int_{\lambda_{1}}^{\lambda_{2}} d \lambda= \pm \operatorname{ctg} \alpha \cdot\left(\lambda_{2}-\lambda_{1}\right) \quad m=\text { ctg } \alpha=\text { const. }
$$

And finally using Equation (E1):

$$
\ln \left[\tan \left(\frac{\phi_{2}}{2}+\frac{\pi}{4}\right)\right]-\ln \left[\tan \left(\frac{\phi_{1}}{2}+\frac{\pi}{4}\right)\right]= \pm \operatorname{ctg} \alpha \cdot\left(\lambda_{2}-\lambda_{1}\right)
$$

This leads to a constant course or bearing between two points connected by a Loxodrome on Mercator chart:

$$
\pm \tan \alpha=\frac{\lambda_{2}-\lambda_{1}}{M_{2}-M_{1}}=\frac{1}{m} \quad M(\phi)=\bar{\psi}(\phi)=\ln \left[\tan \left(\frac{\phi}{2}+\frac{\pi}{4}\right)\right]
$$

Here, $M$ 's are called meridional parts (mer-parts) for respective latitudes (Williams, 1950). Loxodromic distance can be easily calculated using the knowledge of differential geometry (Lipshutz, 1969; Struik, 1988). Employing Equation (A10), the arc-length of Loxodrome part becomes:

$$
L_{1-2}=R \int_{\phi_{1}}^{\phi_{2}}\left[1+\left(\cos ^{2} \phi\right) \cdot\left(\frac{d \lambda}{d \phi}\right)^{2}\right]^{1 / 2} d \phi=R \cdot\left|\frac{\phi_{2}-\phi_{1}}{\cos \alpha}\right|=R \cdot\left|\phi_{2}-\phi_{1}\right| \cdot|\sec \alpha|
$$

Since, $\left(\frac{d \lambda}{d \phi}\right)= \pm \frac{\tan \alpha}{\cos \phi}= \pm \tan \alpha \cdot \sec \phi$, where $0 \leq \alpha<\frac{\pi}{2}$.

As the TC angle (measured from TN) approaches $90^{\circ}$ (but not $90^{\circ}$ itself) the Loxodrome length sharply increases. Kos et al. (1999) obtained similar result utilizing co-latitudes and using similar differential geometry arguments. However, by not using absolute value of co-latitude differences their Loxodrome distance can become negative. Alexander (2004) derived Loxodrome distance using: 


$$
L_{1-2}=R \int_{\phi_{1}}^{\phi_{2}} \sqrt{1+\frac{1}{\psi^{2}(\phi)}\left(\frac{d \lambda}{d \bar{\psi}} \frac{d \bar{\psi}}{d \phi}\right)^{2}} d \phi=R \cdot\left|\phi_{2}-\phi_{1}\right| \cdot|\sec \alpha|
$$

where from Equation (E1):

$$
\frac{d \bar{\psi}}{d \phi}=\sec \phi=\psi(\phi) \quad \frac{d \lambda}{d \bar{\psi}}=\tan \alpha
$$

A practical expression for constant TC (bearing) rhumb line navigation between two points on spherical Earth is now:

$$
\alpha= \pm \tan ^{-1}\left\{\left(\lambda_{2}-\lambda_{1}\right) / \ln \left[\frac{\tan \left(\phi_{2} / 2+\pi / 4\right)}{\tan \left(\phi_{1} / 2+\pi / 4\right)}\right]\right\}
$$

One has to be careful when the Loxodrome is crossing Greenwich antimeridian or approximately the International Date Line (IDL). Conformal Mercator chart can be easily constructed by using $X$ and $Y$ coordinates as (Struik, 1988):

$$
X=\xi \cdot \bar{R}_{E} \cdot\left(\lambda-\lambda_{0}\right) \quad Y=\xi \cdot \bar{R}_{E} \cdot \ln \left[\tan \left(\frac{\phi}{2}+\frac{\pi}{4}\right)\right]
$$

Loxodromes on an ellipsoidal Earth can be also easily calculated in which case the stretching has to include the eccentricity (Alexander, 2004; Bennett, G. G., 1996; Williams, 1950). The latitudes of the corresponding mer-parts are:

$$
\phi(M)=2 \cdot \tan ^{-1}[\exp (M)]-\frac{\pi}{2}
$$

Although the Loxodrome is a curve spiraling infinitely around the SP and the NP, the distance is always finite except for angle of $90^{\circ}(\cos \pi=0)$ :

$$
L_{S P-N P}=\left|\frac{R \cdot \pi}{\cos \alpha}\right| \approx\left|\frac{10,800}{\cos \alpha}\right| \quad[N M] \quad 0 \leq \alpha \leq \pi
$$




\section{Appendix $F$}

\section{SEQM to WMKK route printout}

DATE: 20161213

TIME: 08:42:40

Air Navigation Solutions - Orthodromes and Loxodromes on spherical Earth ver 7.2

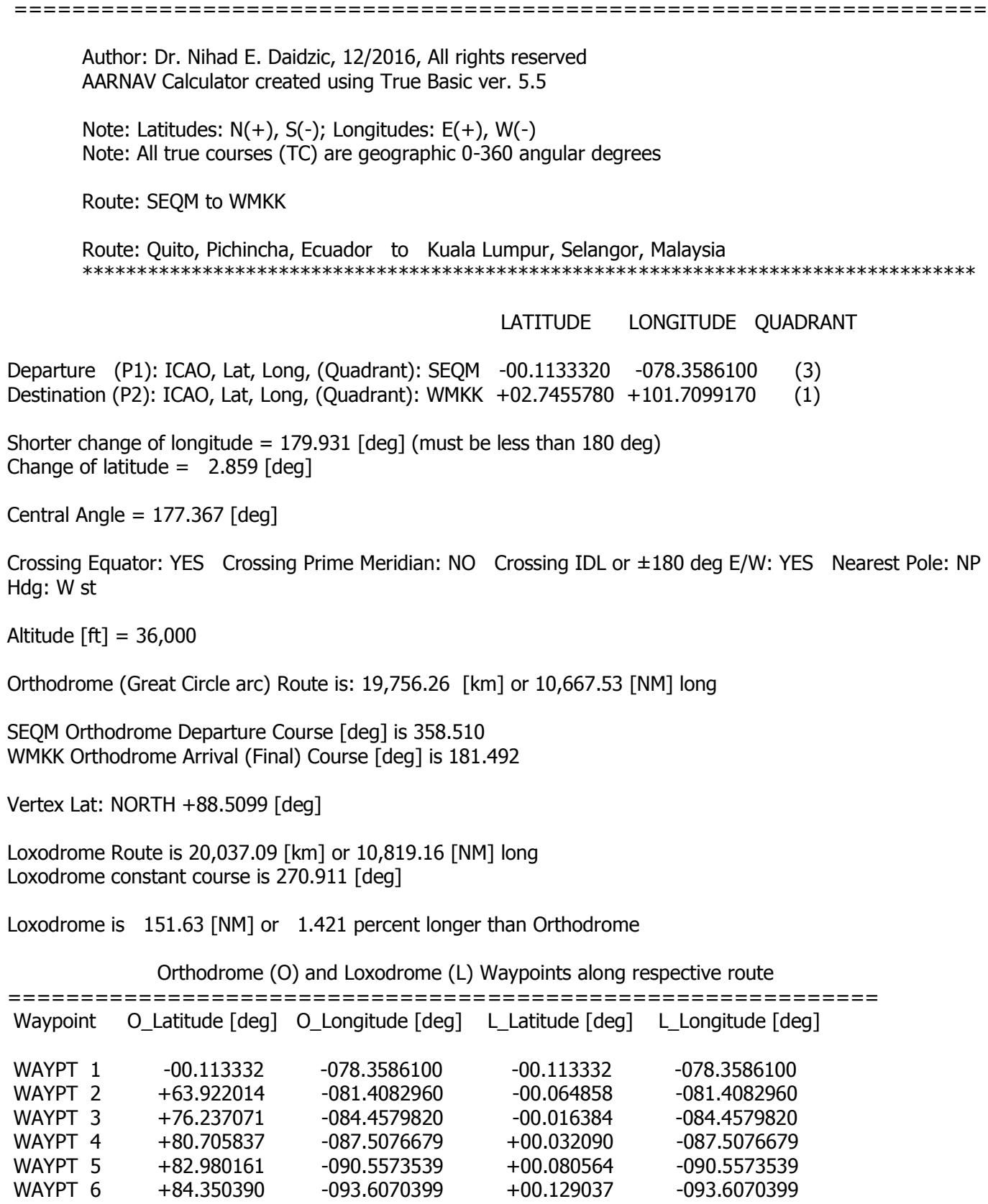




\begin{tabular}{|c|c|c|c|c|}
\hline WAYPT 7 & +85.262914 & -096.6567259 & +00.177511 & -096.6567259 \\
\hline WAYPT 8 & +85.912150 & -099.7064119 & +00.225985 & -099.7064119 \\
\hline WAYPT 9 & +86.396131 & -102.7560979 & +00.274458 & -102.7560979 \\
\hline WAYPT 10 & +86.769589 & -105.8057838 & +00.322932 & -105.8057838 \\
\hline WAYPT 11 & +87.065458 & -108.8554698 & +00.371405 & -108.8554698 \\
\hline WAYPT 12 & +87.304732 & -111.9051558 & +00.419877 & -111.9051558 \\
\hline WAYPT 13 & +87.501416 & -114.9548418 & +00.468350 & -114.9548418 \\
\hline WAYPT 14 & +87.665206 & -118.0045278 & +00.516822 & -118.0045278 \\
\hline WAYPT 15 & +87.803019 & -121.0542138 & +00.565294 & -121.0542138 \\
\hline WAYPT 16 & +87.919918 & -124.1038997 & +00.613765 & -124.1038997 \\
\hline WAYPT 17 & +88.019689 & -127.1535857 & +00.662236 & -127.1535857 \\
\hline WAYPT 18 & +88.105213 & -130.2032717 & +00.710706 & -130.2032717 \\
\hline WAYPT 19 & +88.178716 & -133.2529577 & +00.759176 & -133.2529577 \\
\hline WAYPT 20 & +88.241941 & -136.3026437 & +00.807646 & -136.3026437 \\
\hline WAYPT 21 & +88.296264 & -139.3523297 & +00.856115 & -139.3523297 \\
\hline WAYPT 22 & +88.342780 & -142.4020156 & +00.904583 & -142.4020156 \\
\hline WAYPT 23 & +88.382367 & -145.4517016 & +00.953050 & -145.4517016 \\
\hline WAYPT 24 & +88.415727 & -148.5013876 & +01.001517 & -148.5013876 \\
\hline WAYPT 25 & +88.443421 & -151.5510736 & +01.049983 & -151.5510736 \\
\hline WAYPT 26 & +88.465896 & -154.6007596 & +01.098449 & -154.6007596 \\
\hline WAYPT 27 & +88.483498 & -157.6504456 & +01.146913 & -157.6504456 \\
\hline WAYPT 28 & +88.496493 & -160.7001315 & +01.195377 & -160.7001315 \\
\hline WAYPT 29 & +88.505071 & -163.7498175 & +01.243840 & -163.7498175 \\
\hline WAYPT 30 & +88.509355 & -166.7995035 & +01.292302 & -166.7995035 \\
\hline WAYPT 31 & +88.509406 & -169.8491895 & +01.340763 & -169.8491895 \\
\hline WAYPT 32 & +88.505226 & -172.8988755 & +01.389223 & -172.8988755 \\
\hline WAYPT 33 & +88.496754 & -175.9485615 & +01.437683 & -175.9485615 \\
\hline WAYPT 34 & +88.483869 & -178.9982474 & +01.486141 & -178.9982474 \\
\hline WAYPT 35 & +88.466382 & +177.9520666 & +01.534598 & +177.9520666 \\
\hline WAYPT 36 & +88.444030 & +174.9023806 & +01.583054 & +174.9023806 \\
\hline WAYPT 37 & +88.416468 & +171.8526946 & +01.631509 & +171.8526946 \\
\hline WAYPT 38 & +88.383253 & +168.8030086 & +01.679962 & +168.8030086 \\
\hline WAYPT 39 & +88.343826 & +165.7533226 & +01.728415 & +165.7533226 \\
\hline WAYPT 40 & +88.297488 & +162.7036367 & +01.776866 & +162.7036367 \\
\hline WAYPT 41 & +88.243368 & +159.6539507 & +01.825316 & +159.6539507 \\
\hline WAYPT 42 & +88.180375 & +156.6042647 & +01.873765 & +156.6042647 \\
\hline WAYPT 43 & +88.107142 & +153.5545787 & +01.922212 & +153.5545787 \\
\hline WAYPT 44 & +88.021935 & +150.5048927 & +01.970658 & +150.5048927 \\
\hline WAYPT 45 & +87.922543 & +147.4552067 & +02.019102 & +147.4552067 \\
\hline WAYPT 46 & +87.806103 & +144.4055208 & +02.067546 & +144.4055208 \\
\hline WAYPT 47 & +87.668855 & +141.3558348 & +02.115987 & +141.3558348 \\
\hline WAYPT 48 & +87.505772 & +138.3061488 & +02.164427 & +138.3061488 \\
\hline WAYPT 49 & +87.309992 & +135.2564628 & +02.212866 & +135.2564628 \\
\hline WAYPT 50 & +87.071902 & +132.2067768 & +02.261303 & +132.2067768 \\
\hline WAYPT 51 & +86.777629 & +129.1570908 & +02.309738 & +129.1570908 \\
\hline WAYPT 52 & +86.406394 & +126.1074049 & +02.358172 & +126.1074049 \\
\hline WAYPT 53 & +85.925643 & +123.0577189 & +02.406604 & +123.0577189 \\
\hline WAYPT 54 & +85.281364 & +120.0080329 & +02.455034 & +120.0080329 \\
\hline WAYPT 55 & +84.377011 & +116.9583469 & +02.503463 & +116.9583469 \\
\hline WAYPT 56 & +83.021678 & +113.9086609 & +02.551890 & +113.9086609 \\
\hline WAYPT 57 & +80.778931 & +110.8589749 & +02.600314 & +110.8589749 \\
\hline WAYPT 58 & +76.396247 & +107.8092890 & +02.648738 & +107.8092890 \\
\hline WAYPT 59 & +64.463409 & +104.7596030 & +02.697159 & +104.7596030 \\
\hline WAYPT 60 & +02.745578 & +101.7099170 & +02.745578 & +101.7099170 \\
\hline
\end{tabular}

